Linköping Studies in Science and Technology

Dissertation No. 1943

\title{
Effects of Energetic Disorder on the Optoelectronic Properties of Organic Solar Cells
}

Nikolaos Felekidis

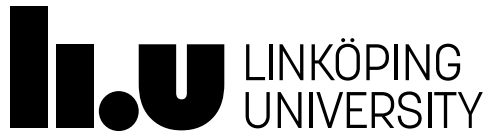

Complex Materials and Devices

Department of Physics, Chemistry and Biology (IFM)

Linköping University, SE-581 83 Linköping, Sweden 


\section{Cover image}

Artistic view of an exciton creation in a polymer-fullerene blend.

Red and black for the blood and mourning of the Pontian genocide.

Blue and white for the Greek sea and waves.

Copyright (C) Nikolaos Felekidis

Effects of Energetic Disorder on the Optoelectronic Properties of Organic Solar Cells

ISSN 0345-7524

ISBN 978-91-7685-271-2

Printed by LiU-Tryck. Linköping, Sweden, 2018 
we have no clue... for now, let's just enjoy the ride 



\section{Abstract}

Organic photovoltaics (OPVs) is a promising low-cost and environmental-friendly technology currently achieving $12-14 \%$ power conversion efficiency. Despite the extensive focus of the research community over the last years, critical mechanisms defining the performance of OPVs are still topics of debate. While energetic disorder is known to be characteristic of organic semiconductors in general, its potential role in OPV has received surprisingly little attention. In this thesis we investigate some aspects of the relation between energetic disorder and several optoelectronic properties of OPV.

Charge carrier mobility is a key parameter in characterizing the performance of organic semiconductors. Analyzing the temperature dependence of the mobility is also an oftenused method to obtain (estimates for) the energetic disorder in the HOMO and LUMO levels of an organic semiconductor material. Different formalisms to extract and analyze mobilities from space charge limited conductivity (SCLC) experiments are reviewed. Surprisingly, the Murgatroyd-Gill analytical model in combination with the Gaussian disorder model in the Boltzmann limit yields similar mobilities and energetic disorders as a more elaborate drift-diffusion model with parametrized mobility functionals. Common analysis and measurement errors are discussed. All the models are incorporated in an automated analysis freeware tool.

The open circuit voltage $\left(\mathrm{V}_{\mathrm{oc}}\right)$ has attracted considerable interest as the large difference between $V_{o c}$ and the bandgap is the main loss mechanism in bulk heterojunction OPVs. Surprisingly, in ternary devices composed of two donors and one acceptor, the $\mathrm{V}_{\mathrm{oc}}$ is not pinned to the shallowest HOMO but demonstrates a continuous tunability between the binary extremities. We show that this phenomenon can be explained with an equilibrium model where $V_{o c}$ is defined as the splitting of the quasi-Fermi levels of the photo-created holes and electrons in a common density of states accounting for the stoichiometry, i.e. the ratio of the donor materials and the broadening by Gaussian disorder. Evaluating the PCE, it is found that ternary devices do not offer advantages over binary unless the fill factor (FF) is increased at intermediate compositions, as a result of improved transport/recombination upon material blending.

Stressing the importance of material intermixing to improve the performance, we found that the presence of an acceptor may drastically alter the mobility and energetic disorder of the donor and vice versa. The effect of different acceptors was studied in a ternary onedonor-two-acceptors system, where the unpredictable variability with composition of the energetic disorder in the HOMO and the LUMO explained the almost linear tunability of $V_{\text {oc. }}$ Designing binary OPVs based on the design rule that the energetic disorder can be 
reduced upon material blending, as we observed, can yield a relative PCE improvement of at least $20 \%$.

CT states currently play a key role in evaluating the performance of OPVs and CTelectroluminescence (CT-EL) is assumed to stem from the recombination of thermalized electron-hole pairs. The varying width of the CT-EL peak for different material combinations is intuitively expected to reflect the energetic disorder of the effective HOMO and LUMO. We employ kinetic Monte Carlo (KMC) CT-EL simulations, using independently measured disorder parameters as input, to calculate the ground-to-ground state (0-0) transition spectrum. Including the vibronic broadening according to the Franck Condon principle, we reproduce the width and current dependence of the measured CT-EL peak for a large number of donor-acceptor combinations. The fitted dominant phonon modes compare well with the values measured using the spectral line narrowing technique. Importantly, the calculations show that CT-EL originates from a narrow, non-thermalized subset of all available CT states, which can be understood by considering the kinetic microscopic process with which electron-hole pairs meet and recombine.

Despite electron-hole pairs being strongly bound in organic materials, the charge separation process following photo-excitation is found to be extremely efficient and independent of the excitation energy. However, at low photon energies where the charges are excited deep in the tail of the DOS, it is intuitively expected for the extraction yield to be quenched. Internal Quantum Efficiency (IQE) experiments for different material systems show both inefficient and efficient charge dissociation for excitation close to the CT energy. This finding is explained by kinetic Monte Carlo simulations accounting for a varying degree of e-h delocalization, where strongly bound localized CT pairs ( $<2 \mathrm{~nm}$ distance) are doomed to recombine at low excitation energies while extended delocalization over 3-5nm yields an increased and energy-independent IQE. Using a single material parameter set, the experimental CT electroluminescence and absorption spectra are reproduced by the same kMC model by accounting for the vibronic progression of the calculated 0-0 transition. In contrast to CT-EL, CT-absorption probes the complete CT manifold.

Charge transport in organic solar cells is currently modelled as either an equilibrium or a non-equilibrium process. The former is described by drift-diffusion (DD) equations, which can be calculated quickly but assume local thermal equilibrium of the charge carriers with the lattice. The latter is described by kMC models, that are time-consuming but treat the charge carriers individually and can probe all relevant time and energy scales. A hybrid model that makes use of the multiple trap and release (MTR) concept in combination with the DD equations is shown to describe both steady-state space charge limited conductivity experiments and non-equilibrium time-resolved transport experiments using a single parameter set. For the investigated simulations, the DD-MTR model is in good agreement with $\mathrm{kMC}$ and $\sim 10$ times faster. 
Steady-state mobilities from DD equations have been argued to be exclusively relevant for operating OPVs while charge carrier thermalization and non-equilibrium time-dependent mobilities (although acknowledged) can be disregarded. This conclusion, based on transient photocurrent experiments with $\mu$ s time resolution, is not complete. We show that non-equilibrium kMC simulations can describe the extraction of charge carriers from subps to $100 \mu$ s timescales with a single parameter set. The majority of the fast charge carriers, mostly non-thermalized electrons, are extracted at time scales below the resolution of the experiment. In other words, the experiment resolves only the slower fraction of the charges, predominantly holes. 



\section{Publications included in the Thesis}

[1] Automated open-source software for reliable charge transport analysis in organic semiconductor diodes

Nikolaos Felekidis, Armantas Melianas, Martijn Kemerink

Organic Electronics (2018), https://doi.org/10.1016/j.orgel.2018.06.010

[2] Open circuit voltage and efficiency in ternary organic photovoltaic blends

Nikolaos Felekidis, Ergang Wang, Martijn Kemerink

Energy \& Environmental Science, 9, 257-266, (2016)

[3] Design Rule for Improved Open-Circuit Voltage in Binary and Ternary Organic Solar Cells

Nikolaos Felekidis, Armantas Melianas, Martijn Kemerink

Applied Materials and Interfaces, 9, 37070-37077, (2017)

[4] Non-Thermal Site-Occupation Governs Charge-Transfer Electroluminescence at Disordered Organic Heterointerfaces

Armantas Melianas, Nikolaos Felekidis, Yuttapoom Puttisong, Luis Ever Aguirre, Stefan C. J. Meskers, René Janssen, Olle Inganäs, Weimin Chen, Martijn Kemerink

Manuscript

[5] Photon Energy Dependence of Long-Range Charge Separation in Organic Bulk Heterojunction Solar Cells

Nikolaos Felekidis, Armantas Melianas, Jonas Bergqvist, Martijn Kemerink Manuscript

[6] Nonequilibrium drift-diffusion model for organic semiconductor devices Nikolaos Felekidis, Armantas Melianas, Martijn Kemerink

Physical Review B, 94, 035205 (2016)

[7] Correspondence on Vincent M. Le Corre et al.

'Charge Carrier Extraction in Organic Solar Cells Governed by Steady-State Mobilities'

Nikolaos Felekidis, Armantas Melianas, Luis Ever Aguirre and Martijn Kemerink Accepted in Advanced Energy Materials 


\section{Contribution to papers}

[1] Prepared the samples, performed all measurements, analyzed and interpreted the data together with the co-authors, performed the fittings, wrote the manuscript draft and revised it with the co-authors.

[2] Prepared the samples, performed all measurements, analyzed and interpreted the data together with the co-authors, developed the equilibrium model together with the supervisor, wrote the manuscript draft and revised it with the co-authors.

[3] Prepared the samples, performed all measurements, analyzed and interpreted the data together with the co-authors, modified the equilibrium model, wrote the manuscript draft and revised it with the co-authors.

[4] Prepared some of the samples, performed some EL-CT and SCLC measurements, performed the SCLC data analysis, helped to write and revise the manuscript.

[5] Prepared the samples, performed most measurements, analyzed and interpreted the data together with the co-authors, performed the simulations, wrote the manuscript draft and revised it with the co-authors.

[6] Prepared the samples, performed all measurements, analyzed and interpreted the data together with the co-authors, performed the simulations, helped with the revision of the manuscript.

[7] Prepared the samples, performed all measurements together with Luis Ever Aguirre, analyzed and interpreted the data together with the co-authors, wrote the manuscript draft and revised it with the co-authors. 


\section{Not included papers}

[1] Photogenerated Carrier Mobility Significantly Exceeds Injected Carrier Mobility in Organic Solar Cells

Armantas Melianas, Vytenis Pranculis, Yuxin Xia, Nikolaos Felekidis, Olle Inganäs, Vidmantas Gulbinas, Martijn Kemerink

Advanced Energy Materials, 7, 1602143, (2017).

[2] Dead ends limit charge carrier extraction from all-polymer bulk heterojunction solar cells

Rokas Jasiūnas, Armantas Melianas, Yuxin Xia, Nikolaos Felekidis, Vidmantas Gulbinas, Martijn Kemerink

Advanced Electronic Materials, (2018), https://doi.org/10.1002/aelm.201800144 



\section{Contents}

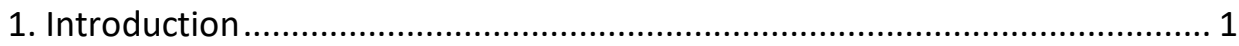

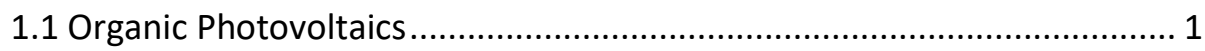

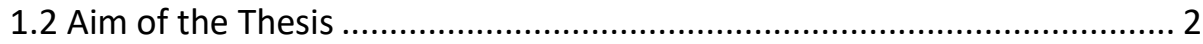

2. Organic Semiconductors...................................................................... 3

2.1 Energetic Disorder ........................................................................... 3

2.2 Charge Carrier Mobility ….................................................................. 5

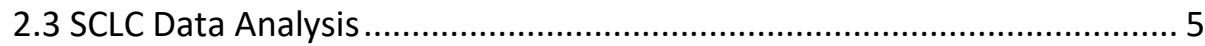

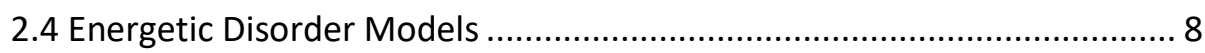

2.5 Automated Analysis Tool .................................................................... 11

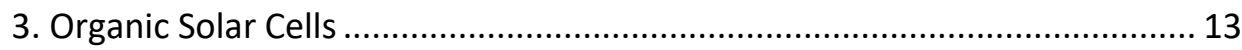

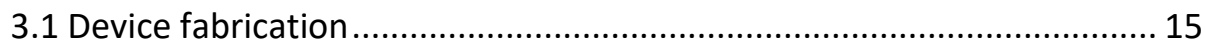

3.2 Electrical and Optical characterization .................................................. 17

3.3 OPV Weight Calculator - Android Application ....................................... 19

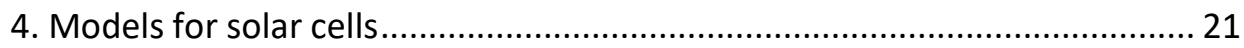

4.1 State-filling quasi-equilibrium model ................................................. 21

4.2 Drift diffusion model with parametrized mobilities ................................ 22

4.3 Kinetic Monte Carlo model .................................................................. 24

4.4 Multiple Trapping and Release + DD model ....................................... 26

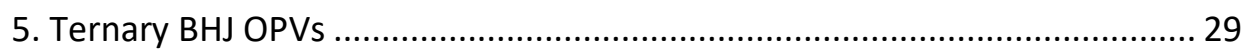

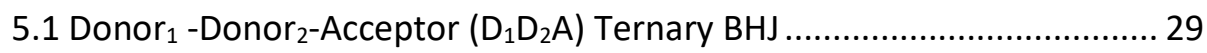

5.2 Donor-Acceptor ${ }_{1}$ Acceptor $_{2}\left(D_{1} A_{2}\right)$ Ternary BHJ................................ 32

6. Optoelectronic processes of CT states …………........................................ 35

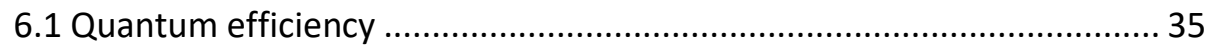

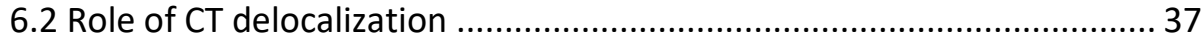

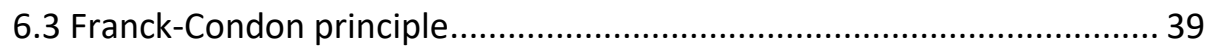

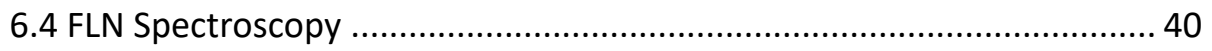


6.5 Origin of CT Electroluminescence......................................................... 41

6.6 Fitting the entire optical spectrum .................................................... 42

6.7 Transient photocurrent experiment limits ........................................... 45

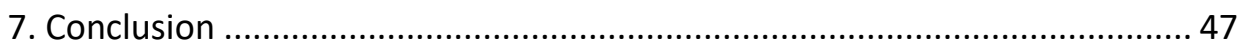

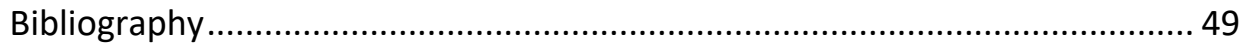

Material abbreviations and chemical structure …........................................ 55

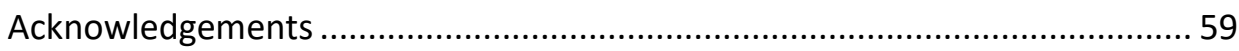




\section{Introduction}

Despite the extended technological progress that followed the industrial revolution, the energy demands of the planet are still mainly satisfied from fossil fuels; a rather limiting and polluting solution due to their finite reserve and $\mathrm{CO}_{2}$ emission. Renewable energy on the other hand is a promising alternative as it is naturally replenished (unlimited) and friendly to the environment. Among the different alternatives (hydropower, wind, biopower, geothermal heat), solar power is an abundant renewable energy source that hits the surface of the earth with an irradiance of $\sim 1000 \mathrm{~W} \mathrm{~m}^{-2}$ and can easily cover the current global electricity demands. Surprisingly, we take very little advantage of this available energy, via the use of photovoltaics (PVs). The etymology of the word reveals its main function: photo (light) is converted to voltage (electric potential difference creating a current). Photovoltaic devices are also commonly referred to as solar cells (SCs).

At the moment, the solar cell market is governed by (inorganic) crystalline silicon, following its dominance in electronics (cheap, stable and non-toxic semiconductor). A solar cell is composed of the absorbing material (active layer), sandwiched between two contacts. Incident photons pass through the (semi-) transparent contact and are absorbed by the active layer. The created bound excitons (excited electron-hole pairs) can overcome their Coulomb binding energy and create free charges that will be collected by the contacts, creating a current in an external circuit. The resulting electrical power can either be directly supplied to a load or stored in batteries/supercapacitors. The theoretical limit for the power conversion efficiency of a silicon solar cell is $\sim 30 \%^{1}$ making the commercially available modules of $\sim 20 \%$ quite an achievement. However, the complexity and cost of manufacturing and installing bulky silicon wafers, makes room for alternatives.

\subsection{Organic Photovoltaics}

Organic solar cells (OSCs) incorporate organic semiconductors (carbon-based polymers and small molecules) as the active layer and offer substantial advantages over their inorganic counterparts. As being non-toxic and processed from solution, organic solar cells can in principle be produced fast, cheap and in large scale using roll-to-roll processing. ${ }^{2}$ Extensive research on new compounds with desirable optoelectronic properties has currently set the power conversion efficiency at $12 \%-14 \%$ for state-of-the-art small scale solar cells prepared in controlled lab conditions. ${ }^{3,4}$ 
Despite the rapid development of organic solar cells over the last decades, this technology is still immature for viable commercialization due to major limiting factors as stability and power conversion efficiency in modules. Ironically, most of the organic semiconductors used in solar cells, photo-degrade rapidly when exposed to ambient conditions under solar illumination. Furthermore, all reported high efficiencies are not yet fully compatible with continuous roll to roll fabrication techniques as the efficiencies of large scale devices are dramatically decreased. ${ }^{5}$

One of the characteristic intrinsic material properties of organic semiconductors is energetic disorder, which is a commonly overlooked material property in OSCs and will be discussed thoroughly in the coming chapters. Investigating the role of energetic disorder will help understand basic mechanisms that affect the performance of OSCs.

\subsection{Aim of the Thesis}

The aim of this work is to examine the role of static energetic disorder on the optoelectronic properties of organic semiconductors. A combination of electrical and optical experiments analyzed with suitable models, provides a deeper understanding of still debatable physical mechanisms within OSCs among which are:

- Measuring and modeling energetic disorder

- Proper analysis of charge transport experiments to extract mobilities and energetic disorder

- The relation of the open-circuit voltage with energetic disorder in organic solar cells

- The role of disorder and delocalization in the charge separation efficiency

- The role of the energetic disorder in the shape the emission and absorption spectra

- Description of transient and steady-state experiments 


\section{Organic Semiconductors}

Organic semiconductors (OSCs) are carbon-based $\pi$-conjugated systems with a bandgap of $\sim 1.4-4 \mathrm{eV}$ and a small dielectric constant (3-4). These promising flexible, light-weight, solution-processed materials (polymers and small molecules) are widely used in organic electronic applications spanning from OSCs to organic light emitting diodes (OLEDs) and organic field effect transistors (OFETs). ${ }^{6,7}$ Organic semiconducting polymers can be designed to have different backbone configurations as well as various incorporated heteroatoms as e.g. $\mathrm{N}$ and $\mathrm{S}$, resulting in practically unlimited design possibilities. It is therefore of importance to evaluate the charge transport efficiency of these materials in a robust and time-effective manner.

\subsection{Energetic Disorder}

The variation of the polymer chain lengths and the appearance of conformational defects (kinks and twists) in the polymer backbone, result in strong variations (disorder) of the site energies. The resulting energetic disorder is a nearly unavoidable material property that is also related to morphological features as polymer/fullerene aggregation and crystallinity while it sets the limit of the relatively poor conductivity/charge carrier mobility in organic semiconductors. A commonly assumed shape for the density of states (DOS) of bulk organic semiconductors is a Gaussian distribution described by:

$$
g(E)=\frac{N_{0}}{\sqrt{2 \pi \sigma^{2}}} \exp \left(-\frac{\left(E-E_{0}\right)^{2}}{2 \sigma^{2}}\right)
$$

where $E$ is the energy of the site, $E_{0}$ is the mean energy, $N_{0}$ is the concentration of sites and $\sigma$ is the standard deviation, as shown in Figure 1. The latter will be referred to as the energetic disorder in this thesis. 


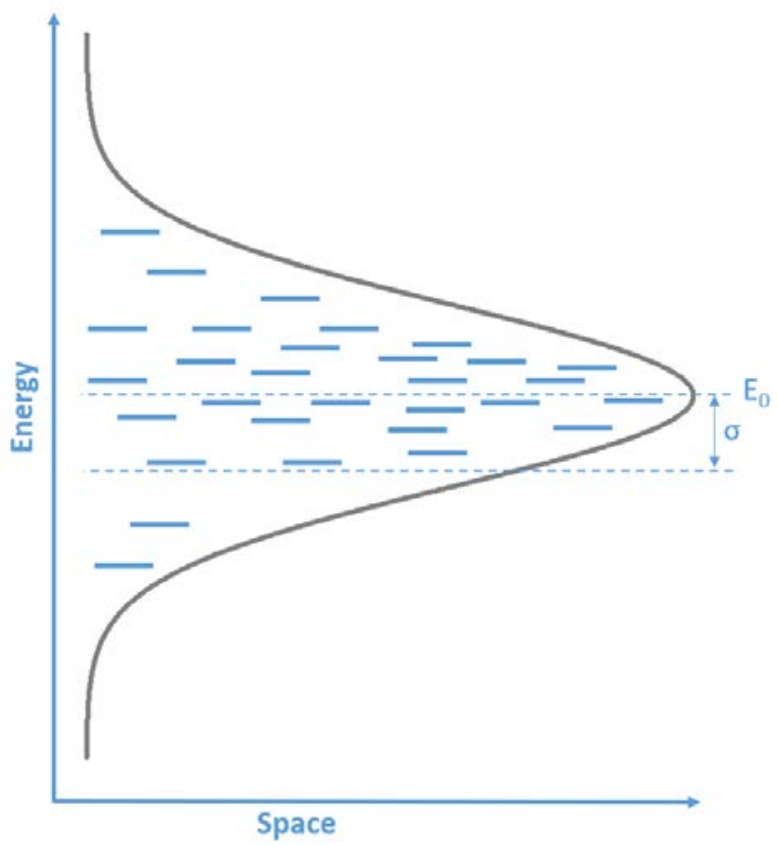

Figure 1. Gaussian distribution of energy sites with mean energy $E_{0}$ and standard distribution $\sigma$.

Different shapes of the DOS can be used as e.g. in organic field-effect transistors, where an exponential distribution is commonly described by:

$$
g(E)=\frac{N_{0}}{k T_{0}} \exp \left(\frac{\mathrm{E}}{k T_{0}}\right), E \leq 0
$$

where $T_{0}$ is the characteristic temperature of the distribution ( $k T_{0}$ is equivalent to the energetic disorder). In this thesis the shape of the DOS is always assumed to be Gaussian, according to equation 1 . 


\subsection{Charge Carrier Mobility}

The charge carrier mobility $\mu$, is an intrinsic material property measured in $\mathrm{m}^{2} \mathrm{~V}^{-1} \mathrm{~s}^{-1}$ that describes the average speed of a charge carrier in the material under the presence of an electric field. Charge transport in OSCs is described as a hopping (thermally activated tunnelling) process between different molecular sites which are distributed by Gaussian energetic disorder (discussed in detail in chapter 4.3). In addition to the electric field, mobility is enhanced by temperature (thermally assisted process) and charge carrier concentration. $^{8-10}$ There are different experimental techniques to measure the charge carrier mobility, each one probing different time scales and having its own complexity and sample preparation demands. Among others, the most popular techniques are timeresolved Terahertz Spectroscopy (TRTS), Electro-Absorption (EA), time-resolved electric field-induced second harmonic generation (TREFISH) probing charge carrier mobilities in time scales from sub-picoseconds to tens of nanoseconds. 'Slower' experimental techniques are time-resolved Microwave Conductance (TRMC), Transient Photocurrent (TPC), Time-of-Flight (TOF) and Charge Carrier Extraction by Linearly Increasing Voltage (CELIV) which can resolve mobilities in the microsecond range while the most common steady-state experiment is space-charge limited conductivity (SCLC).

\subsection{SCLC Data Analysis}

The SCLC experiment is a very simple technique to study charge transport in diode-type semiconductor single carrier devices and allows the extraction of near-equilibrium steadystate temperature dependent mobilities. A voltage is applied to the device injecting charge carriers via the ohmic contacts and the resulting current is measured for single carrier devices (hole- and electron-only). For this type of devices, the contacts are properly selected to have the same workfunction (symmetricity) in alignment with the HOMO/LUMO in which transport will take place (selectivity). Since only holes (electrons) are injected/transported in the HOMO (LUMO), i.e. no recombination is taking place, they will fill up the device creating a space-charge that limits the transport of new charge carriers, resulting in a space-charge limited current. The experiment must be done in absolute dark to ensure that charge transport of the injected carriers is probed.

The simplest analytical expression describing the SCL current density $J$ (measured current divided by the active area) is the quadratic equation by Mott-Gurney: ${ }^{11}$ 


$$
J=\frac{9}{8} \varepsilon_{0} \varepsilon_{r} \mu \frac{\left(V-V_{b i}\right)^{2}}{L^{3}}
$$

where $L$ is the thickness of the active layer, $V$ the applied field, $V_{b i}$ the built-in voltage, $\varepsilon_{0}$ the vacuum permittivity and $\varepsilon_{r}$ the material's relative dielectric constant. It is common to plot the SCLC data in a $\log J-\log V$ scale where the quadratic law of equation 3 translates to a slope of 2 (Figure 2).

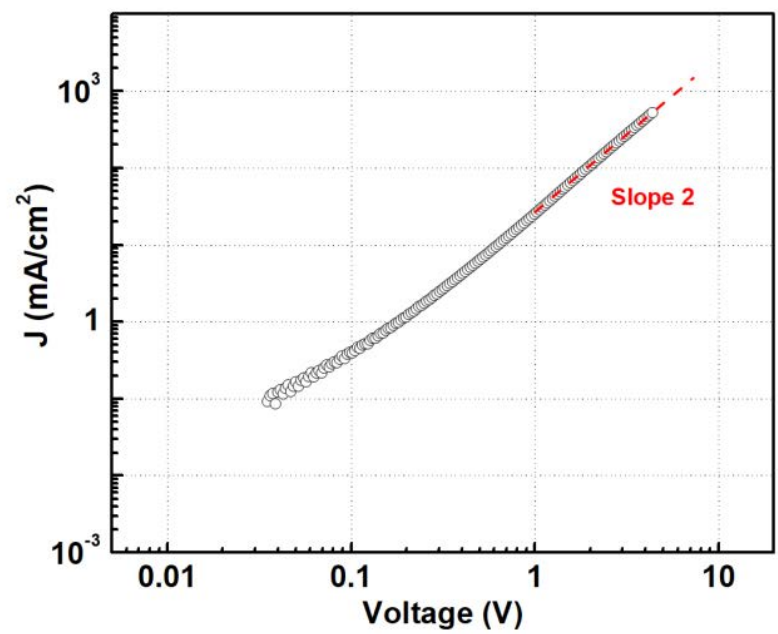

Figure 2. SCLC data (black traces) plotted on a logJ-logV scale having a slope of 2 (red trace) for rrP3HT:PC ${ }_{61} B M$ 1:1 measured at 280K.

Experimental SCLC data commonly have varying slopes, typically larger than 2, due to the presence of traps and energetic disorder. ${ }^{12,13} \mathrm{An}$ extended version of equation 3 that can describe slopes larger than 2, taking into account the enhancement of the mobility in presence of a strong field (Frenkel effect) is: ${ }^{14}$

$$
J=\frac{9}{8} \varepsilon_{r} \varepsilon_{0} \mu_{0} \frac{\left(V-V_{b i}\right)^{2}}{L^{3}} \exp \left(0.891 \gamma \sqrt{\frac{V-V_{b i}}{L}}\right)
$$

where $\mu_{0}$ is the zero-field mobility and $\gamma$ an empirical field enhancement factor. The resulting zero-field mobilities can be analyzed by different models based on different formalisms.

It is demonstrated in paper 1 that (forced) application of equation 3 on datasets where the slope is not 2, will result in poor fits and overestimated mobilities. As part of a proper analysis method, it is also important to select the correct voltage range, avoiding traps and series resistance or tortuosity, by examining the slope of the double log JV data. The existence of traps is observed as a distinct peak in slope (trap filling regime) at lower 
voltages while a decreasing slope (smaller than 2, described by a negative $\gamma$ ) at high voltages and temperature, is a sign of series resistance or tortuosity. ${ }^{15}$ The fitting voltage region must be shifted to higher and lower voltages respectively to ensure that transport in the bulk of the material is correctly probed, as shown in Figure 3.
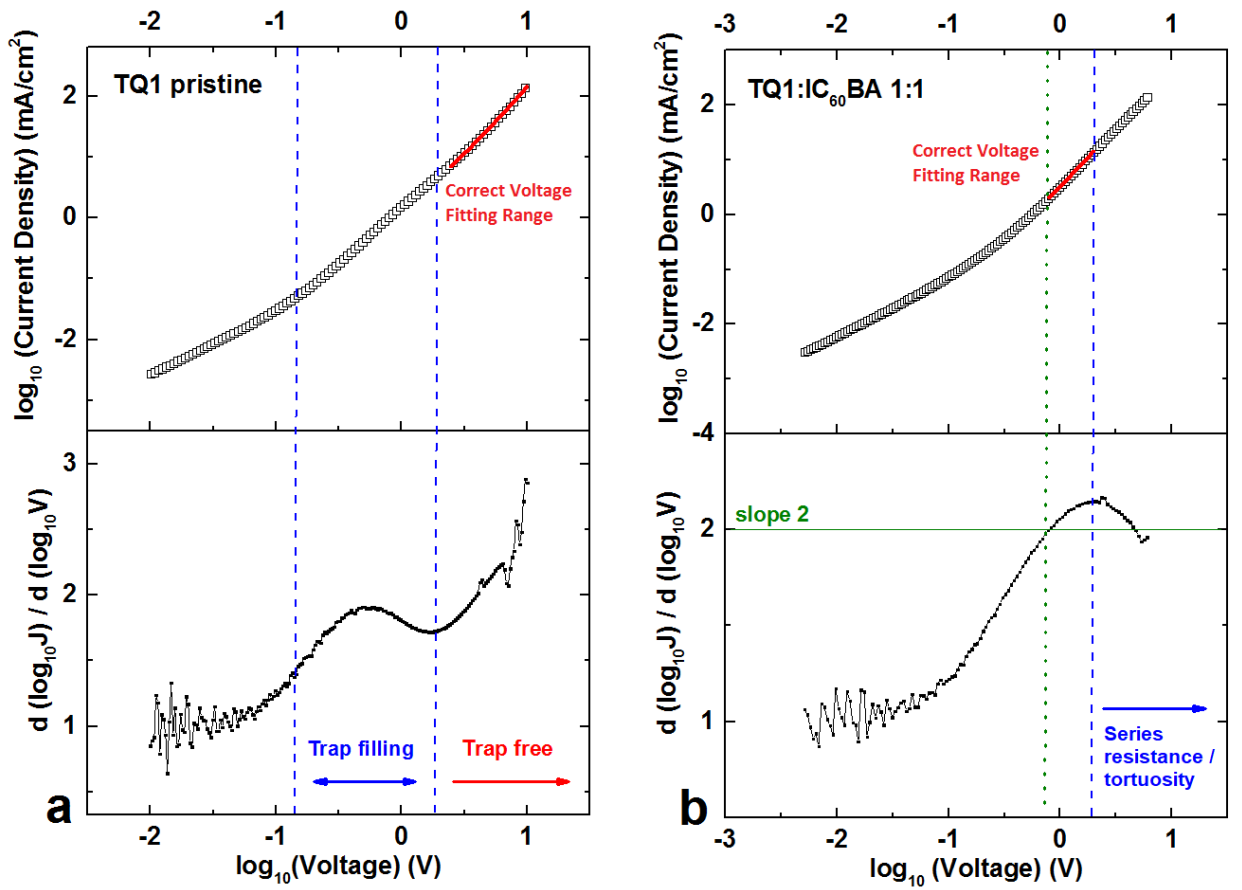

Figure 3. a) (top) SCLC data (black squares) measured at $300 \mathrm{~K}$ for a pristine TQ1 hole-only device, showing a peak in the slope of the double-log $J-V$ curve as a sign of a trap-filling regime (bottom). b) Same for a binary TQ1:IC $\mathrm{C}_{60} \mathrm{BA}$ 1:1 hole-only device showing non-negligible series resistance or tortuosity effects by a decrease in the J-V slope at higher voltages. Reproduced from Organic Electronics (2018) with permission from Elsevier.

The SCLC JV measurements can be performed in high vacuum for temperatures typically ranging between $200 \mathrm{~K}-300 \mathrm{~K}$ and estimates of the energetic disorder can be extracted with any of the available energetic disorder models. 


\subsection{Energetic Disorder Models}

\subsubsection{Gaussian Disorder Model}

The temperature dependence of the charge carrier mobilities can be used to extract the energetic disorder according to different formalisms. A simple analytical model is the Gaussian Disorder Model (GDM) in which the zero-field mobility follows a non-Arrhenius dependence on the temperature $\left(\mu_{0} \sim 1 / T^{2}\right)$ according to: ${ }^{16}$

$$
\mu_{0}(T)=\mu^{*} \exp \left(-c\left(\frac{\sigma}{k T}\right)^{2}\right)
$$

where $\mu^{*}$ is the infinite temperature mobility and $c$ is a constant, set to $\left[\frac{2}{3}\right]^{2}=0.44 . .^{10}$

\subsubsection{Extended Gaussian Disorder Model}

This simple analytical GDM model does not account for the dependence of the mobility on charge carrier concentration and applied field. A more advanced version is called the Extended GDM (eGDM) model in which the mobility is parametrized to the lattice constant $a_{N N}$ (average distance between neighbouring sites placed on a 3D grid), attempt frequency $v_{0}$ (a hopping rate from site to site representing the phonon frequency and transfer integral) and the Gaussian disorder $\sigma$, according to: ${ }^{10}$

$$
\mu(T, p)=\frac{a_{N N}^{2} v_{0} q}{\sigma} c_{1} \exp \left(-c_{2} \widehat{\sigma}^{2}\right) \exp \left(\frac{1}{2}\left(\widehat{\sigma}^{2}-\widehat{\sigma}\right)\left(2 p a_{N N}^{3}\right)^{\delta}\right)
$$

where $\hat{\sigma}=\sigma / k T$ is the reduced disorder and $p$ is the charge carrier concentration. The parameters $c_{1}$ and $c_{2}$ are set equal to 0.87 and 0.44 respectively (for more details see paper 1). The field dependence is included as

$$
\mu(T, p, E)=\mu(T, p) \exp \left(0.44\left(\hat{\sigma}^{1.5}-2.2\right)\left(\sqrt{1+0.8 E_{\text {red }}^{2}}-1\right)\right)
$$

where $E_{\text {red }}=E q a_{N N} / \sigma$ is the reduced field. More details on the equations are found in paper 1 for this model, in which the energetic disorder is uncorrelated. The existence of a DOS shape together with the parametrized mobility expressions make the specific model suitable for kinetic Monte Carlo (kMC) and drift diffusion (DD) simulations, which are extensively used in the thesis, as described in chapter 4. 


\subsubsection{Correlated Gaussian Disorder Model}

A variation of the eGDM model accounts for the presence of molecular dipoles which can give rise to spatial correlations in the energy distribution of the sites (correlated GDM). The general expression describing the temperature, field and charge carrier concentration in this model is: ${ }^{17}$

$$
\mu(T, p, E)=\left[\left(\mu_{\text {low }}(T, p, E)\right)^{q(\widehat{\sigma})}+\left(\mu_{\text {high }}(T, p, E)\right)^{q(\widehat{\sigma})}\right]^{1 / q(\widehat{\sigma})}
$$

where $q(\hat{\sigma})=2.4 /(1-\hat{\sigma})$ and $\mu_{\text {low }} / \mu_{\text {high }}$ are the mobilities in the low- / high-field regimes. Full equations are found in paper 1.

\subsubsection{Effective temperature model}

Another variation of the model, describes the effect of a finite applied field via an increased effective temperature according to: ${ }^{18}$

$$
T_{e f f}=\left[T^{2}+\left(\gamma \frac{q E \alpha}{k_{B}}\right)^{2}\right]^{1 / 2}
$$

where $\gamma \approx 0.67, k_{B}$ the Boltzmann constant and the localization length $\alpha$ is the characteristic length scale, equal to $0.1 \mathrm{~nm}$ (paper 1 ). In this work, it was combined with the general mobility equation derived from 'fat' percolation: ${ }^{19,20}$

$$
\mu(T, p)=B \frac{q \omega_{0}}{k T a_{N N} p}\left(\frac{k T}{\sigma}\right)^{-\lambda} \exp \left(\frac{E_{F}(T, p)-E^{*}}{k T}\right)
$$

where $E_{F}$ is the Fermi level, $E^{*}=-0.491 \sigma$ is the critical energy and $\lambda$ is the critical exponent of the correlation length of the percolation cluster, set equal to $0.875 .{ }^{20}$ 


\subsubsection{Arrhenius type model}

Opposed to the GDM model (where $\left.\mu_{0} \sim 1 / T^{2}\right)$, an Arrhenius type $\left(\mu_{0} \sim 1 / T\right)$ analytical model describes the zero-field mobility as: ${ }^{21}$

$$
\mu_{0}(T)=\mu^{*} \exp \left(-\frac{\Delta}{k T}\right)
$$

with $\Delta$ the activation energy. In this model, no assumptions are made for the shape of the DOS. A universal infinite temperature mobility $\mu^{*}$ was argued for a series of pristine holeonly materials, the existence of which would reproduce the full temperature dependence of the mobility with a single measurement. In paper 1 , this universality is tested for a wide range of material systems (pristine, binary and ternary) where $\mu^{*}$ is found to vary significantly, i.e. no strict universal law was detected. 


\subsection{Automated Analysis Tool}

All the models mentioned in the previous sections are implemented in an automated freeware analysis tool. ${ }^{22}$ The software allows the user to easily fit the SCLC data and extract mobilities and Gaussian disorder values, by selecting any of the analytical or drift-diffusion models. A graphical user interface (GUI) allows fast and easy data analysis requiring minimum input from the user (Figure 4).

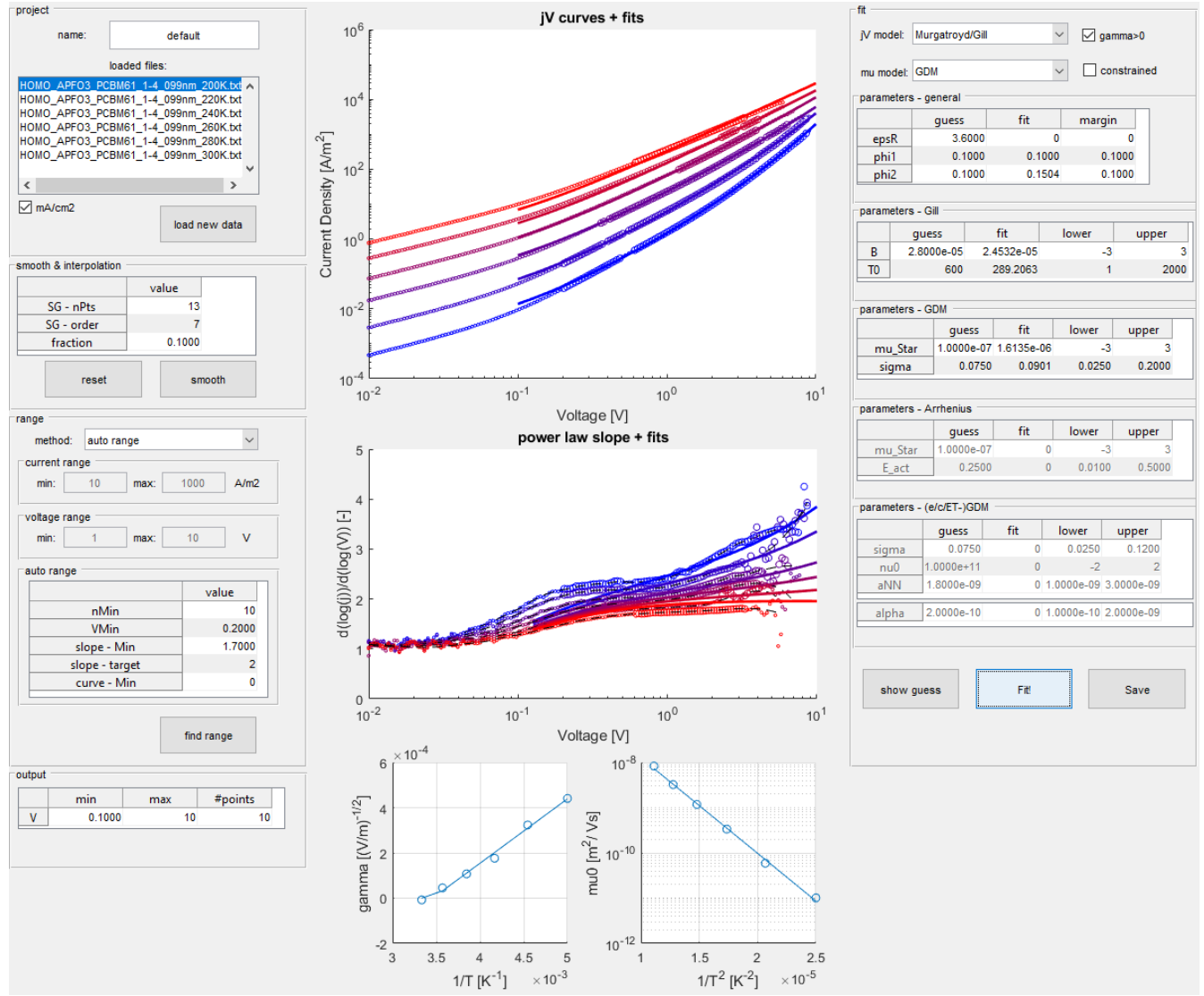

Figure 4. Graphical user interface of SCLC automated fitting tool. Temperature-dependent SCLC data for hole-only APFO3:PC ${ }_{61} B M$ 1:4 are fitted with the MG and GDM models. 
This automated analysis tool was implemented to analyze hole-only SCLC data for pristine, binary and ternary hole-only devices (paper 1). The use of the eGDM model for the parametrization of the mobility combined with DD transport equations, resulted in excellent description of the experimental data. This is in consistency with kMC simulations, in which the parameters from the eGDM model are also used to describe the energetics and the hopping rates, successfully reproducing optoelectronic experiments (papers 4-7). This agreement further justifies the selection of the eGDM formalism for the dependence of the mobility on the charge carrier concentration, applied electric field and temperature.

The software is available as a Matlab library or as a standalone executable file for free at: https://github.com/mkemerink/FitSCLC 


\section{Organic Solar Cells}

Organic solar cells are devices that convert light to electricity based on the properties of organic semiconductors. They commonly consist of a transparent metallic contact which allows light to enter the cell and be absorbed in the photo-active layer (PAL), i.e. the semiconductor. The incident photon energy is absorbed and promotes an electron from the Highest Occupied Molecular Orbital (HOMO) to the Lowest Occupied Molecular Orbital (LUMO) leaving back a hole. Hence only photons with energy larger that the optical gap (HOMO-LUMO energy difference) will be absorbed and create excitons. The electron-hole pair needs to be split and collected at the contacts to create an electric current. Typically, the workfunction of the hole-collecting contact (anode) is selected appropriately to match the HOMO of the semiconductor. The same holds for the workfunction of the electroncollecting contact (cathode) and the LUMO of the semiconductor. The second contact is usually made of a reflective metal to increase the absorption from the back-reflected light.

Solar cells incorporating only one organic semiconductor material as the active layer, are doomed to fail as the strong exciton Coulomb binding energy in organic materials ( $\sim 0.5$ $1 \mathrm{eV}$ ) cannot be overcome by the available thermal and field energy of a typical OSC device at room temperature. The solution to this problem is the introduction of a second semiconductor (called the acceptor) with a LUMO level lying deeper than the one of the donor. The working principle is that an excited electron in the absorber (donor) will favour its transportation to the available states in the LUMO of the acceptor, which are lower in energy. This will physically separate the electron-hole pair, creating a charge-transfer (CT) state, facilitating their dissociation (Figure 5). The separated charge carriers can use the available excitation energy to escape to the contacts assisted by the built-in field from the contacts $\left(\sim 10^{7} \mathrm{~V} / \mathrm{m}\right)$ and the available thermal energy ( $25 \mathrm{meV}$ at room temperature). 


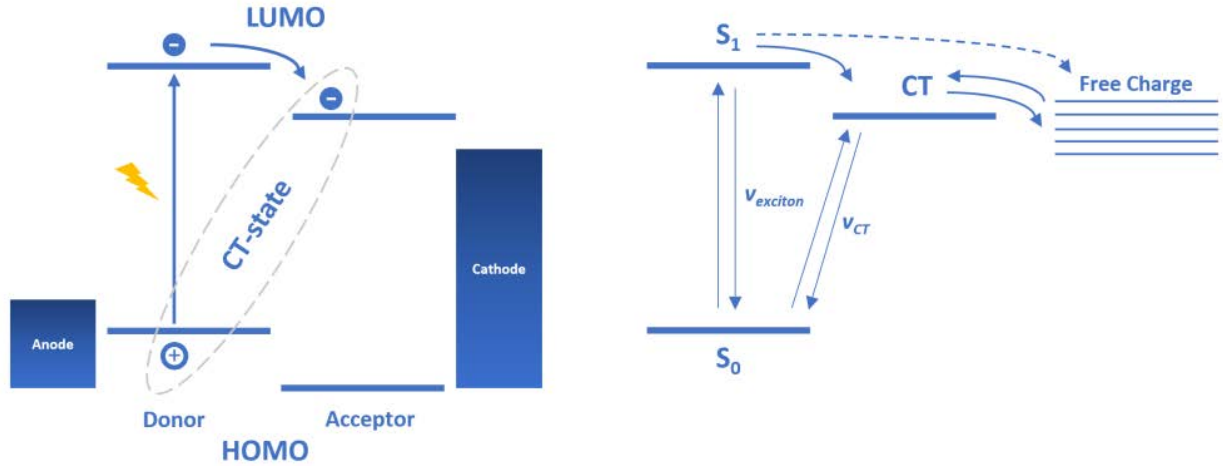

Figure 5. (left) Simplified band diagram showing the creation of a CT-state across the donor-acceptor interface following photo-excitation. (right) Jablonski diagram showing the pathways for exciton/CT creation and recombination as well as free charge generation.

The simplest configuration of a donor and an acceptor is a bilayer (two layers on top of each other) where the excitons can split at the interface and travel to the contacts through the two materials. This design has the disadvantage that only the bilayer interface participates in the creation of free electrons and holes hence it is not a very efficient configuration. It is a powerful tool though for studying OSCs and is still used for research purposes. Opposed to the idea of a bilayer, mixing the two materials together in a blend of specific stoichiometry, thus creating a bulk-heterojunction ( $\mathrm{BHJ}$ ) active layer, proved to be very effective as the increased number of D-A interfaces contribute to the generation of free electron-hole pairs. Both configurations are shown in Figure 6, where the active layer is sandwiched between a semi-transparent and a reflecting contact.
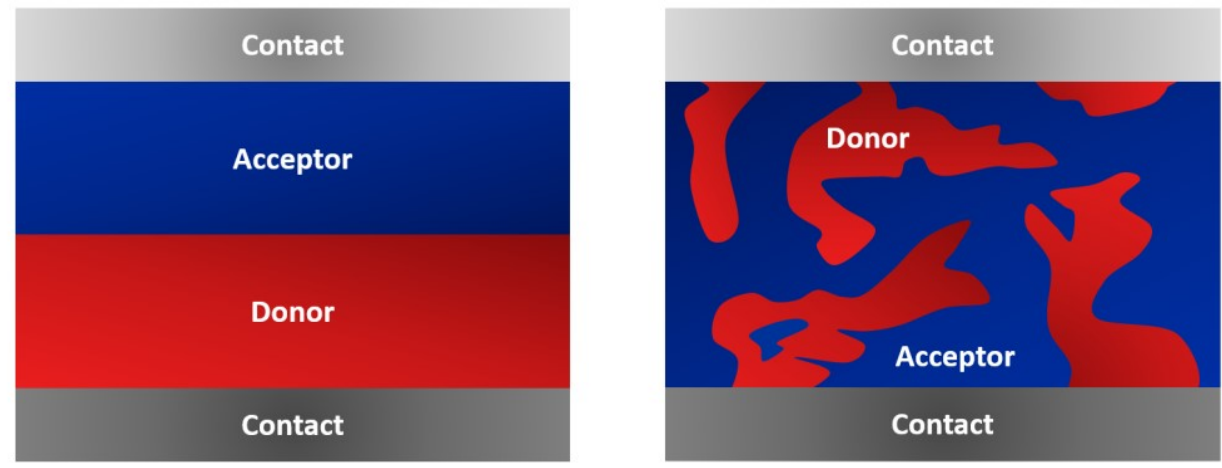

Figure 6. Acceptor-Donor bilayer (left) and BHJ (right) PAL configuration sandwiched between a semitransparent top and a reflective bottom contact. 


\subsection{Device fabrication}

The fabrication of organic solar cell devices involves many steps as each layer requires special treatment. Most of the steps take place in controlled environment, i.e. a glovebox filled with nitrogen where the oxygen/water concentration is <1ppm to avoid material degradation.

The first step is to make the photo active layer solution using the proper solvent and having the desired/optimal concentration as well as Donor:Acceptor ratio. The solution is steered on a hot plate at a temperature below the boiling point of the solvent (usually 4-5 hours are enough, although solutions are commonly steered overnight to create a well-dissolved solution). According to the conventional device architecture, glass substrates with a layer of ITO (typically thicker than 100nm) are cleaned with detergent and TL1 and then PEDOT:PSS 4083 is spin coated at 3000 rpm for 40s in ambient, creating a thin film of $40 \mathrm{~nm}$. This interlayer modifies the workfunction of ITO to make it a low workfunction contact. The active layer is then spin coated in the glovebox with spin speeds depending on the solvent and the desired thickness (typically $\sim 500 \mathrm{rpm}$ for chlorobenzene and $\sim 1500 \mathrm{rpm}$ for chloroform with PAL thicknesses typically varying from 70 to $130 \mathrm{~nm}$ for high performance OSCs). At this point the device may be annealed to modify the morphology and enhance the performance; this process depends mainly on the active layer materials. The final step is to evaporate the electron accepting contact in high vacuum (a common interlayercontact combination is $0.6 \mathrm{~nm} \mathrm{LiF}-90 \mathrm{~nm}$ Al. The last fabrication step is to encapsulate the devices with precleaned transparent glass stripes using UV glue, minimizing oxidation upon ambient exposure. This is the main process (with small variations) that was followed in the fabrication of all OSCs in this thesis (Figure 7).

\begin{tabular}{|c|}
\hline Encapsulation Glass \\
\hline Aluminum \\
\hline LiF \\
\hline Active Layer \\
\hline PEDOT:PSS \\
\hline ITO \\
\hline Glass \\
\hline
\end{tabular}

Figure 7. Conventional OSC device architecture. Incoming photons enter the device from the bottom semi-transparent ITO contact and are reflected by the top Al contact. 
Single carrier devices are fabricated by selecting the interlayer-contact material/thickness combination appropriately, in order to have equal workfunctions, i.e. two hole/electron selective contacts and allowing only the respective charge carriers to be transported in the device. Typical combinations are ITO/PEDOT:PSS-MoO$/ \mathrm{Al}$ for hole-only devices and ITO/ZnO-LiF/Al for electron-only devices. Single carrier devices are usually fabricated to investigate charge transport and energetics of the HOMO/LUMO of pristine materials and blends. As part of this thesis, hole- and electron-only devices were fabricated using the contact combinations mentioned above.

It is important to point out the major deviation of the current fabrication methods in the lab compared to the targeted realistic roll-to-roll processing techniques that will take advantage of the solution processability and large scale, low-cost manufacturing of organic solar cells. All the high efficiencies reported for organic solar cells refer to small scale devices prepared in controlled environment with evaporated contacts and are measured directly after production, usually in the glovebox. However, attempts to transfer the smallscale lab results to large scale roll-to-roll processed devices in ambient conditions, results in a dramatic decrease of the performance of the solar cells. ${ }^{5}$ 


\subsection{Electrical and Optical characterization}

\subsubsection{JV under illumination}

The performance of a solar cell is characterized by measuring the current density as a function of the applied voltage $(J-V)$ under constant $\sim 1$ Sun illumination. The varying applied voltages are the equivalent representation of different loads connected to the device, effectively acting as voltage sources of opposite polarity, reducing the total output voltage of the solar cell. A typical J-V curve under illumination is shown in Figure 8.

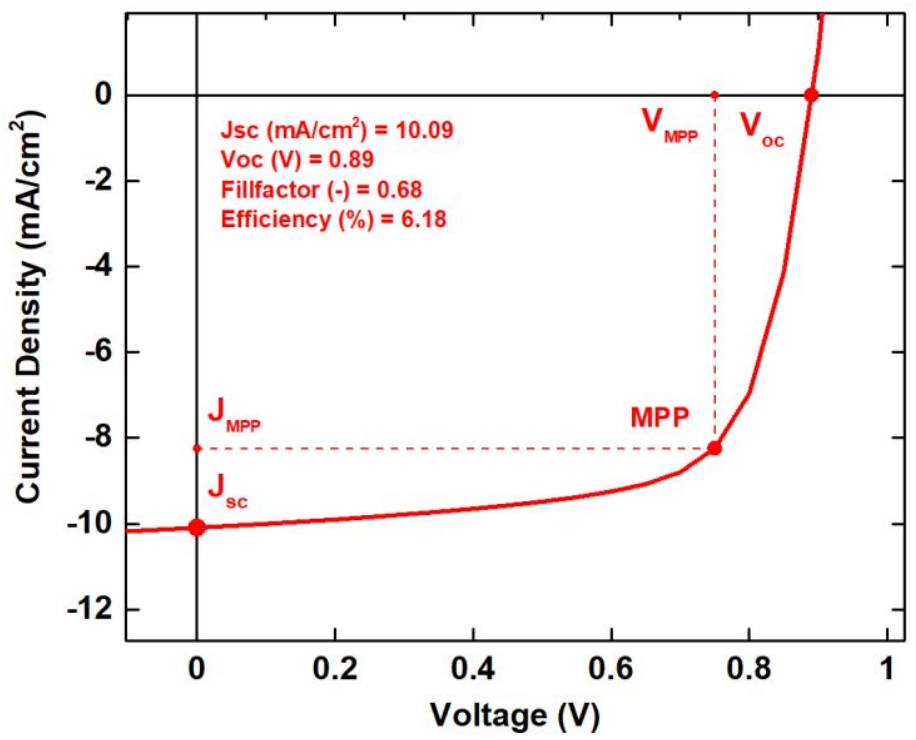

Figure 8. JV under $\sim$ 1Sun illumination for TQ1: $\mathrm{PC}_{71} \mathrm{BM}$ 1:2.5 measured at room temperature

When no voltage is applied $(\mathrm{V}=0)$ the device operates in the presence of a zero-resistance load (short circuit) and the effective field in the device is the built-in field (from the difference of the contact workfunctions), resulting in the short-circuit current $\left(\mathrm{J}_{\mathrm{sc}}\right)$. Similarly, the maximum output voltage is measured when the current density is zero and it is called open-circuit voltage $\left(\mathrm{V}_{\mathrm{oc}}\right)$. In these operating conditions, the field in the device as a result of the contacts' workfunction difference is counterbalanced by the external applied field and no current flows in the device (equivalent to a connected load with infinite resistance, i.e. an open circuit). Both $J_{s c}$ and $V_{o c}$ are limiting cases that deliver no output power $\left(P_{\text {out }}\right.$ ) as $P_{\text {out }}=J \cdot V$. In real operating conditions there is a maximum power point $P_{\max }$ $=\mathrm{J}_{\mathrm{Pmax}} \cdot \mathrm{V}_{\mathrm{Pmax}}$ where the device should optimally operate (Figure 8). The ratio of the optimum 
power and the maximum theoretical power $\left(\mathrm{JPmax}_{\mathrm{max}} \cdot \mathrm{V}_{\mathrm{Pmax}} / \mathrm{J}_{\mathrm{sc}} \cdot \mathrm{V}_{\mathrm{oc}}\right)$ is a measure of the ideality of the device and is called fill factor (FF). The PCE of the solar cell is defined as the ratio of the output to the input solar power (AM 1.5 solar spectrum has an irradiance of $1000 \mathrm{~W} / \mathrm{m}^{2}$ ) and is calculated as:

$$
P C E=\frac{P_{\text {out }}}{P_{\text {in }}}=\frac{V_{\text {oc }} \cdot J_{s c} \cdot F F}{P_{\text {in }}}
$$

\subsection{2 $V_{o c}$ and CT energy}

$V_{\text {oc }}$ describes the energy of the extracted holes and electrons and is therefore proportional to the CT gap, i.e. the difference between the acceptor LUMO and the donor HOMO. A common method of estimating the CT energy ( $\left.E_{C T}\right)$ is to extract it as the intersection point of the CT emission and absorption spectra ${ }^{23}$ as shown in Figure 9 and discussed in detail in chapters 6.5 and 6.6.

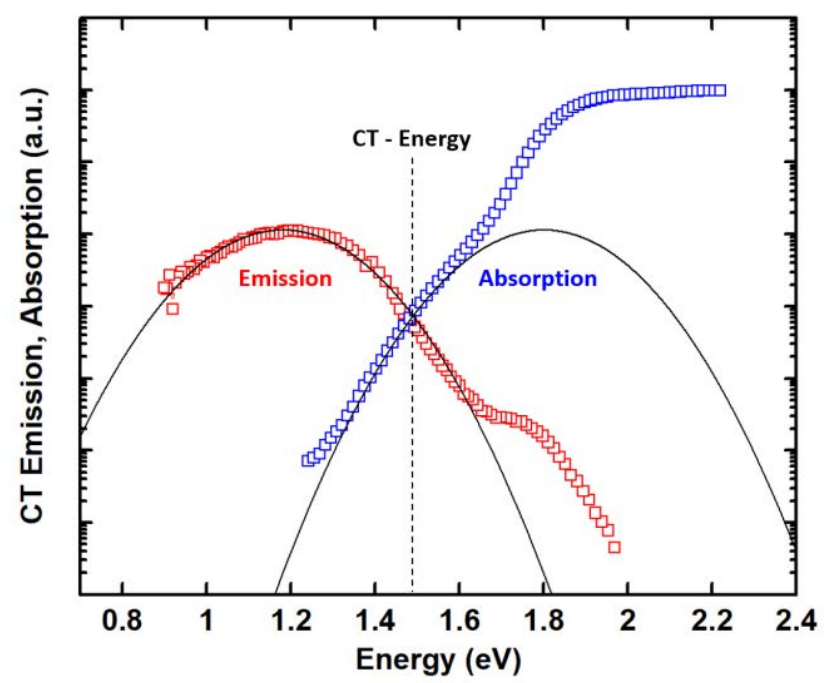

Figure 9. $\mathrm{CT}$ energy taken as the intersection point of $\mathrm{CT}$ absorption (blue trace) and CT emission (red trace) spectrum. The spectra are scaled using shifted Gaussians of the same magnitude.

The $V_{o c}$ is calculated as:

$$
V_{o c}=\frac{E_{C T}}{q}+\frac{k T}{q} \ln \left(\frac{J_{S c} h^{3} c^{2}}{f q 2 \pi\left(E_{C T}-\lambda\right)}\right)+\frac{k T}{q} \ln \left(E Q E_{E L}\right)
$$

where $f$ is a measure of the donor-acceptor coupling, $\lambda$ is the reorganization energy and $E Q E_{E L}$ is the emission external quantum efficiency. The second (third) term of equation 13 represents radiative (non-radiative) energy losses. ${ }^{23}$ 


\subsection{OPV Weight Calculator - Android Application}

When the active layer solution is being prepared, the calculated weight for the donor and acceptor materials must be weighed on a precision scale to achieve the desired D:A ratio and solution concentration. This is not always trivial, especially with soft light-weighed materials that are difficult to handle precisely, which makes it typical for the weighed amount to deviate from the desired value. As the extracted and weighed material should never be returned to the source container, it is common to use the whole amount and update the solvent volume as well as the weight of the rest of the material(s), keeping the desired ratio and solution concentration constant. An automated android application has been developed as part of this thesis, that ensures flawless and quick recalculation of the updated material weights and solvent volume. ${ }^{24}$ In specific, the application is a simple active layer weight calculator for binary and ternary material systems (discussed in chapter 5) that can be carried in the lab and used on the spot. The user can set the desired ratio, concentration and solvent volume via the user interface (Figure 10) and the application will automatically calculate the required weight of the components. The most convenient feature of this tool is the fact that the user can update the measured weight (having a deviating value) and the application will automatically update the solvent volume and the weight of the rest of the materials, keeping the ratio and the active layer concentration constant (which are both vital during device fabrication).

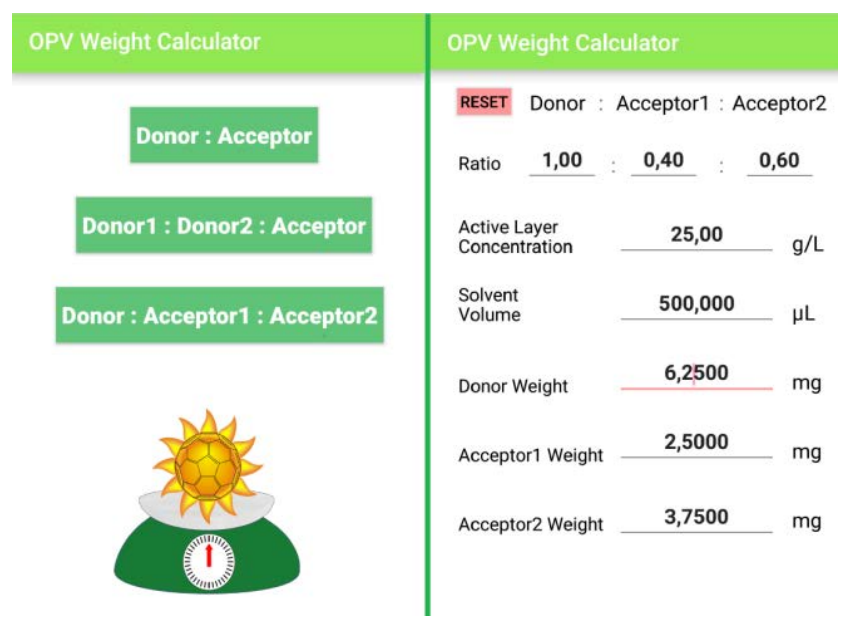

Figure 10. Available modes of operation (left) and user interface for data entry (right).

The application is available for free for Android devices and can be found in Google Play as 'OPV Weight Calculator' or downloaded directly from: 


\section{Models for solar cells}

Modeling of OSCs is a vital research tool, complementary to experiments, attracting significant scientific focus. Many basic principles defining the performance of OSCs are still subject of debate and the underlying mechanisms can be studied by simulations. There are different kinds of models available according to their complexity: analytical and phenomenological models are based on closed expressions to describe physical phenomena which makes them easy and fast to implement. ${ }^{11,14,25}$ On the other hand, stochastic/numerical models predict probability distributions based on random variations of selected parameters thus being more demanding to implement and more timeconsuming to execute despite the increasing computational power. ${ }^{16,26,27}$ There can also be hybrid models combining the best features of each of the two aforementioned categories, i.e. closed expressions in combination with random numerical calculations, as discussed later in this chapter. Four different models have been implemented as part of this thesis to study different mechanisms in single-carrier devices and organic solar cells.

\subsection{State-filling quasi-equilibrium model}

A quasi-equilibrium state filling model was developed to explain and understand the surprising experimental $\mathrm{V}_{\mathrm{oc}}$ behavior in ternary BHJ OSCs as discussed in papers 2 and 3. Ternary OSCs incorporate two donors or two acceptors and are discussed in detail in chapter 5 . The model assumes that charge carriers are in (quasi) thermal equilibrium with the lattice enabling the use of (quasi) Fermi levels to describe their energy. The DOS is assumed to follow a Gaussian distribution and the presence of two materials is represented by a joint DOS, i.e. the sum of the two individual DOS where the stoichiometry of the ternary blend is the weighing factor. By selecting the Gaussian energetic disorder $\sigma$, the HOMO-LUMO levels of the materials and the occupation, i.e. the fraction of occupied states (DOOS/DOS), the model calculates the quasi-Fermi level for the electrons and holes. The $V_{o c}$ is defined as their difference, as shown in Figure 11. Adding a second donor with a shallower HOMO level will shift the Fermi level to higher energies, in order to maintain the same occupation (constant illumination) in the new joint DOS, reducing the $\mathrm{V}_{\text {oc. }}$. Based on this simple mechanism of state filling, the surprising behaviour of the $V_{o c} v$ donor concentration was explained for $\mathrm{D}_{1} \mathrm{D}_{2} \mathrm{~A}$ ternary blends, as discussed in chapter 5 . 

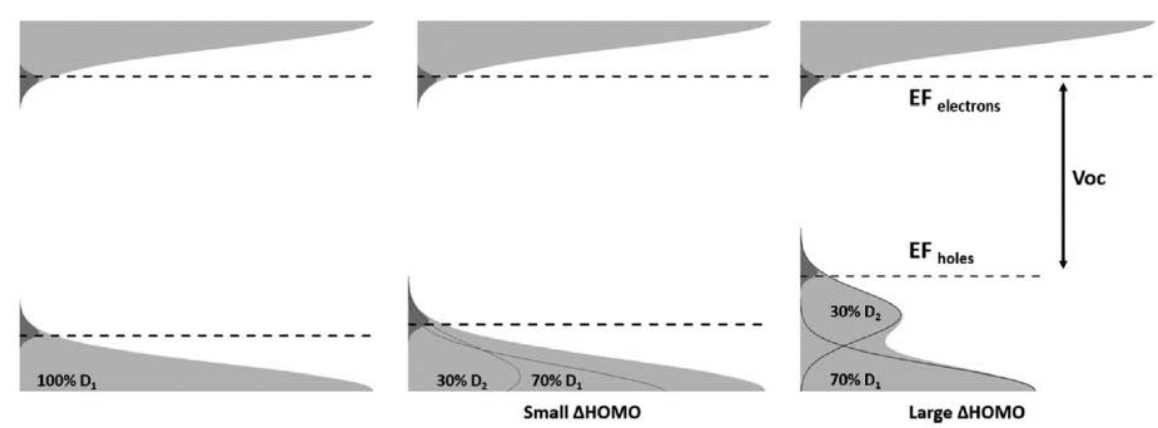

Figure 11. Joint DOS for different donor stoichiometry. The $\mathrm{V}_{\mathrm{oc}}$ (arrow) is defined as the difference between the electrons and holes quasi-Fermi level. Reproduced from Energy \& Environmental Science, 9, 257-266 (2016) with permission from The Royal Society of Chemistry

A simple optical model in which the absorption spectrum of the donors is corrected for their stoichiometry, calculates the flux of charge carriers under illumination as:

$$
\dot{n}=\dot{p}=\frac{1}{L_{\text {device }}} \int \frac{A M 1.5(\lambda) \cdot I Q E(\lambda) \cdot A b s_{\text {Donors }}(\lambda)}{E_{p h}(\lambda)} d \lambda
$$

where $L_{\text {device }}$ is the active layer thickness, $A b s_{\text {Donors }}(\lambda)$ is the absorption spectrum of the donors and $E_{p h}(\lambda)$ is the photon energy. The short-circuit current $j_{s c}$ is calculated as:

$$
j_{s c}=q \cdot \dot{p} \cdot L_{\text {device }}
$$

The PCE can then be predicted using the experimental FF and equation 12 , evaluating the effect of energetic disorder and ternary donor compositions, as discussed in chapter 5.

\subsection{Drift diffusion model with parametrized mobilities}

A more physical approach to modeling OSCs as full devices, i.e. active layer and contacts is based on drift-diffusion (DD) equations. In this quasi-equilibrium model, the charge carrier concentration as a function of the (one dimensional) position in the device is described by the Poisson equation:

$$
\nabla^{2} V(x)=-\frac{\rho(x)}{\varepsilon_{0} \varepsilon_{r}}
$$

where $\rho$ is the charge carrier density, $V$ the electrostatic potential, $\varepsilon_{0}$ the vacuum permittivity, $\varepsilon_{r}$ the relative dielectric constant of the material and $x$ is the one-dimensional 
position in the device. The DD equation for the current density of holes and electrons is described by:

$$
\begin{aligned}
& j_{p}=-q p \mu_{p} \frac{\partial}{\partial x} V-q D_{p} \frac{\partial}{\partial x} p \\
& j_{n}=-q n \mu_{n} \frac{\partial}{\partial x} V+q D_{n} \frac{\partial}{\partial x} n
\end{aligned}
$$

where $\mu_{p(n)}$ is the hole (electron) mobility, $p(n)$ is the hole (electron) charge carrier density and $D_{p(n)}$ is the hole (electron) diffusion constant. The diffusion constant is calculated from the Einstein relation $D=\mu k T / q$. The continuity equations for holes and electrons are:

$$
\begin{aligned}
& \frac{\partial}{\partial t} p=G-R_{p}+\frac{1}{q} \cdot \frac{\partial}{\partial x} j_{p} \\
& \frac{\partial}{\partial t} n=G-R_{n}+\frac{1}{q} \cdot \frac{\partial}{\partial x} j_{n}
\end{aligned}
$$

where $G$ is the generation rate of charge carriers and $R_{p(n)}$ the recombination rate of holes (electrons). At steady-state $\frac{\partial}{\partial t} p$ and $\frac{\partial}{\partial t} n$ are set equal to 0 .

In such models the mobility can be a function of the temperature, field and charge carrier concentration. In this thesis the extended Gaussian disorder model is implemented, as described in chapter 2.4, where the mobility is parametrized to the lattice constant $\alpha_{N N}$, attempt to hop frequency $v_{0}$ and Gaussian energetic disorder $\sigma$ (equation 6). This DD+eGDM model can calculate $J-V$ curves in seconds hence it can be combined with a leastsquares fitting algorithm using $\alpha_{N N}, v_{0}$, and $\sigma$ as fitting parameters.

In paper 1 charge carrier mobilities and energetic disorder are extracted from temperature dependent SCLC experiments on hole-only diodes using this model. Surprisingly, the analytical Murgatroyd-Gill (MG) expression (equation 4) is found to extract similar mobilities to the more advanced DD+eGDM model for a wide number of hole-only material systems. The corresponding Gaussian disorder values are in agreement between eGDM and MG+GDM for low fullerene concentrations as shown in Figure 12. 


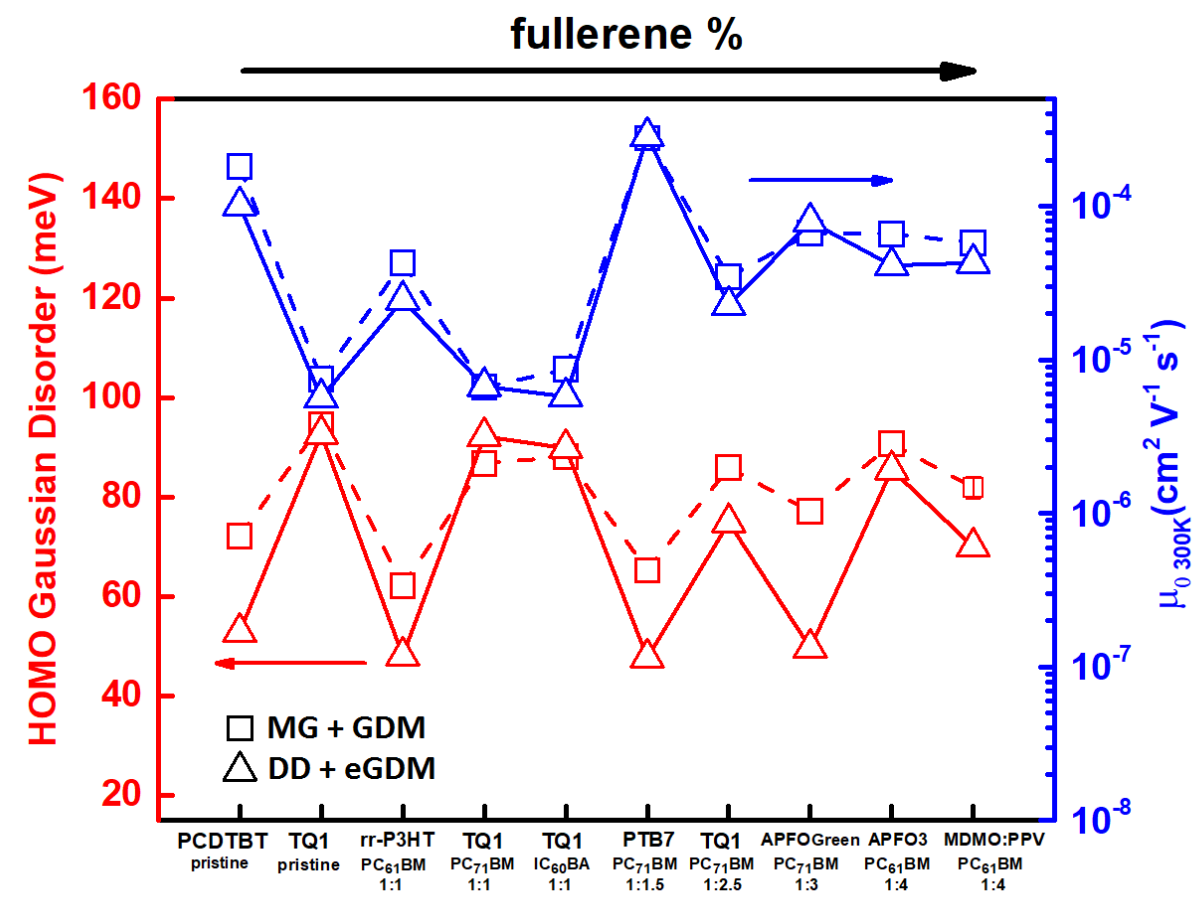

Figure 12. Zero-field mobilities and energetic disorder for MG+GDM and DD+eGDM models. Reproduced from Organic Electronics (2018) with permission from Elsevier

\subsection{Kinetic Monte Carlo model}

Despite their advantages, quasi-equilibrium models cannot describe transient phenomena and are not suitable to study thermalization and dispersion in organic disordered semiconductors. ${ }^{26}$ Stochastic kinetic Monte Carlo models where the motion of charge carriers is simulated at a particle level allows access to the energetics at any time scale, i.e. from early non-equilibrium events up to (quasi) equilibrium steady state time scales. As part of the present thesis, a kinetic Monte Carlo model (developed by Martijn Kemerink) was implemented and used extensively in papers 4-7. In this numerical model all charges are treated individually allowing for detailed characterization of charge carrier photocreation, injection, transport, recombination and extraction as a function of energy, space and time; the occurrence of the above events is determined by random numbers. The model assumes a cubic lattice (grid) with a defined inter-site distance $\left(\alpha_{N N}\right)$. Transport is described as a thermally activated tunnelling process between neighboring sites (nearest neighbor hopping NNH) according to the Miller-Abrahams expressions for the hopping rate: ${ }^{28}$ 


$$
v_{i f}=\left\{\begin{array}{cc}
v_{0} \exp \left(-\frac{E_{f}-E_{i} \pm q \vec{r}_{i f} \cdot \vec{F}+\Delta E_{C}}{k_{B} T}\right)= & v_{0} \exp \left(-\frac{\Delta E_{i f}}{k_{B} T}\right)\left(\Delta E_{i f}>0\right) \\
v_{0} & \left(\Delta E_{i f} \leq 0\right)
\end{array}\right.
$$

In the above equation $\vec{F}$ is the external electric field and $\vec{r}_{i f}$ the vector connecting initial (i) and final sites $(f) . \Delta E_{C}$ is the Coulomb energy variation calculated explicitly from interactions of all charges in the device as well as the image charges with the moving charge carrier. The above equation describes the reduced probability of a charge carrier to climb up in energy while hops to lower energies take place with the maximum rate $v_{0}$. This is illustrated in Figure 13.

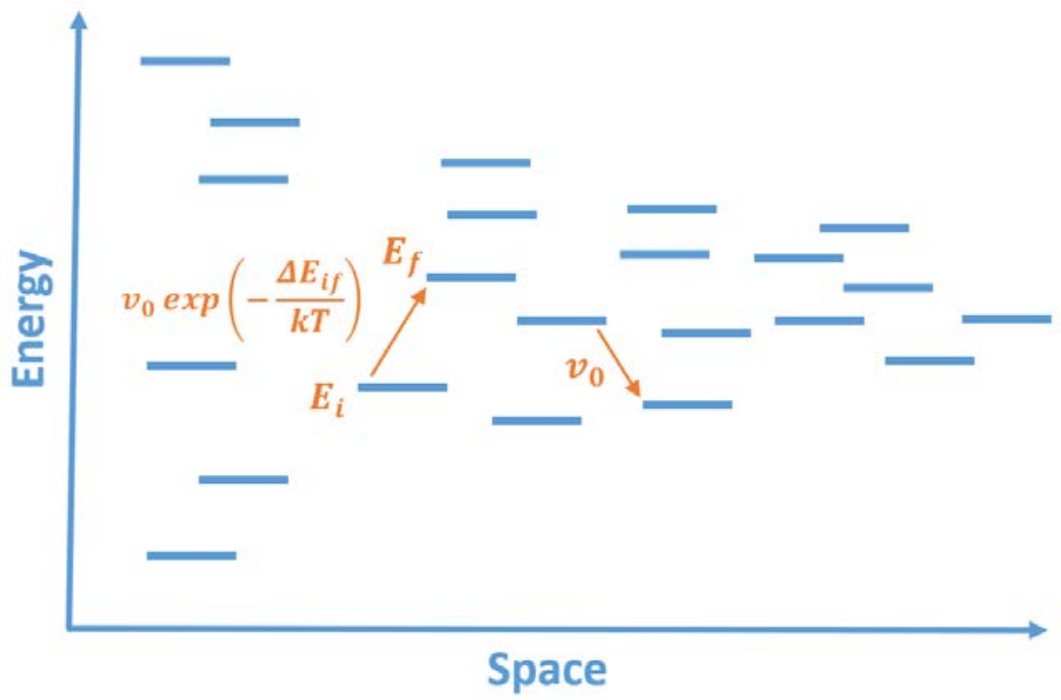

Figure 13. Nearest neighbour hopping rates according to Miller-Abrahams formalism

The bulk heterojunction is treated as an effective medium, i.e. charges move in the HOMO (LUMO) of the donor (acceptor) with hopping rate $v_{0}$ and Gaussian disorder $\sigma$ parameters.

This model is a powerful analysis tool that provides insight by examining the energetics of holes and electrons in different transient (non-equilibrium) and steady state (quasiequilibrium) experiments, as will be shown in chapter 6 . This complex model is time consuming and many independent configurations need to be averaged, making it nonsuitable for least-squares fitting routines. 


\subsection{Multiple Trapping and Release + DD model}

In an attempt to bridge the gap between simple/fast equilibrium and complex/slow numerical models, a kinetic-DD model was developed based on the multiple trapping and release (MTR) ${ }^{29}$ mechanism in combination with DD equations. This model can be thought of a simple and faster version of the kinetic Monte Carlo model described in the previous chapter, where charge transport does not happen as a series of hops from site to site but after one critical hop from the Fermi level to the mobility edge; this is effectively the transport level above which the charge carriers are allowed to move into the device (as shown in Figure 14) with a free mobility and a traveling distance given by:

$$
\begin{gathered}
\mu^{*}=\frac{v_{0} \cdot \alpha_{N N}^{2}}{\sigma_{D O S}} \\
\Delta z=\mu^{*} F t_{\text {free }} \pm \sqrt{2 D^{*} t_{\text {free }}}
\end{gathered}
$$

where $D^{*}=\mu^{*} k_{B} T$. The rate at which charge carriers are excited from trap (localized) states to the transport level (mobility edge) is given by:

$$
v_{1}=v_{0} \exp \left(-\frac{E_{c}-E_{\text {trap }}}{k_{B} T}\right)
$$

while trapping takes place with rate $v_{0} . t_{\text {free }}$ is the time a charge carrier moves in the band between two trapping events, calculated as $t_{\text {free }}=-\ln X / v_{0}$, where $X$ is a random number between 0 and 1. 


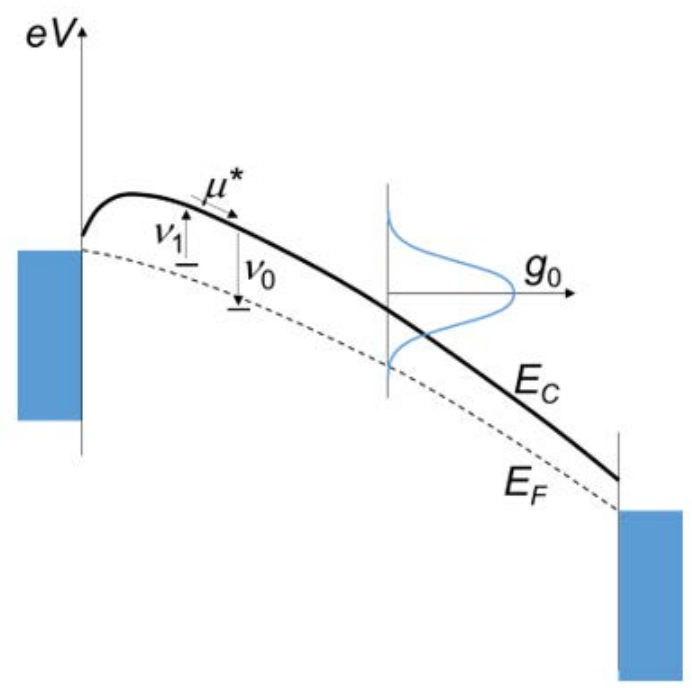

Figure 14. Schematic depiction of the (de)trapping and transport mechanism for the MTR model. Reproduced from Physical Review B, 94, 035205 (2016) with permission from the American Physical Society

This model (developed by Martijn Kemerink) reproduces transient and steady-state charge transport experiments of hole-only devices in agreement with $\mathrm{kMC}$ simulations (paper 6). 


\section{Ternary BHJ OPVs}

Conventional BHJ OSCs are composed of two organic semiconducting materials in a blend (binary systems), however it is quite common to introduce more materials to increase their performance and stability. As part of this thesis, the effect of introducing a third organic semiconductor, thus creating a ternary (composed of three materials) blend, is studied. The motivation behind the selection of two donors or two acceptors is different, as will be discussed in the next two chapters.

\subsection{Donor 1 -Donor 2 -Acceptor $\left(D_{1} D_{2} A\right)$ Ternary $B H J$}

As commonly donors are the main absorbers in polymer-fullerene organic solar cells, the idea behind the use of two donors in such material systems is to increase the absorption spectral coverage. Mixing materials with complementary absorption spectra will increase the photocurrent and hence the PCE. However, the increase in photocurrent by introducing a second donor with smaller bandgap (red-shifted absorber), will result in a reduction of the voltage which is proportional to the difference between the acceptor LUMO and the (effective in this case) donor HOMO. This mechanism is shown in Figure 15.

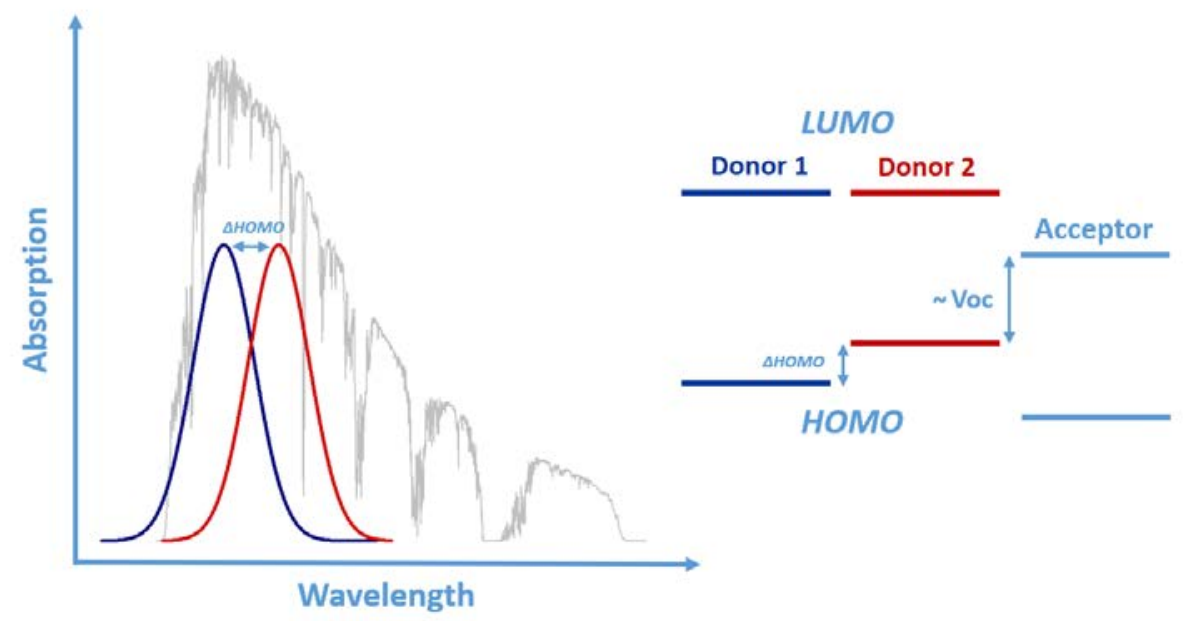

Figure 15. Complementary absorption spectra for two donor ternary systems (left) and band diagram showing the voltage limitation due to the shallower HOMO (right).

It is therefore of great interest to investigate whether the interplay between current and voltage can be ultimately beneficial for the performance of ternary OPVs. Another surprising feature is the composition dependence of the $V_{o c}$ in a $D_{1}(x): D_{2}(1-x): A, 0 \leq x \leq 1$ 
ternary system, as it is not proportional to the difference between the shallowest donor HOMO and the acceptor LUMO. In thermal equilibrium, all holes would be expected to thermalize to the shallowest HOMO thus pinning the $\mathrm{V}_{\text {oc }}$ to the smallest possible value. On the contrary, the $V_{o c}$ is found to be composition-dependent, i.e. tunable between the binary $D_{1} A$ and $D_{2} A V_{o c}$ values. ${ }^{30-32}$ The explanation to this phenomenon is still under debate. A "parallel junction" model was proposed in which the different donor materials act independently as distinct binary cells. ${ }^{33,34}$ Since they are unavoidably connected via the contacts, the two materials cannot act individually and the lowest voltage photo-diode will always short the highest one. An alternative model, in which the blended materials are proposed to form an alloy, i.e. a perfect blend acting as one material with highly delocalized wavefunctions, ${ }^{35}$ seems unlikely for inhomogeneous blends of different polymers mixed with fullerene molecules. A cascade of energy levels, facilitating the escape of electrons and holes has also been proposed for efficient ternary blends, however the fact that the Voc should be pinned to the highest HOMO is not discussed. ${ }^{36}$ In this approach the $\mathrm{D}_{1}: \mathrm{D}_{2}$ :Acceptor HOMO (LUMO) levels will be in ascending (descending) order, 'protecting' the extraction of the charge carriers against recombination. However, it is not clear how an unfavourable alignment is avoided, where the material with the shallowest HOMO (deepest LUMO) will end up in the middle of the blends, effectively trapping the charge carriers and reducing the $\mathrm{V}_{\mathrm{oc}}$.

The surprising behavior of $\mathrm{V}_{\mathrm{oc}}$ was confirmed in our work, for two different sets of material systems $\mathrm{TQ}_{\mathrm{m} 6}: \mathrm{TQ}_{\mathrm{m} 12}: \mathrm{PC}_{71} \mathrm{BM}$ and $\mathrm{TQ}_{\mathrm{p} 6}: \mathrm{TQ}_{\mathrm{m12}}: \mathrm{PC}_{71} \mathrm{BM}$ (see Appendix for molecular structure) where the donors have a small and large HOMO difference respectively (paper 2). The resulting $V_{o c}$ vs stoichiometry is composition-dependent, in-between the values of the binaries and has a large (small) variation following the big (small) HOMO level difference of the polymer donors as shown in Figure 15 . The dependence of $V_{\text {oc }}$ on the donor composition was explained using the state-filling model introduced in chapter 4.1 which is in good agreement with the experimental findings (Figure 16). 


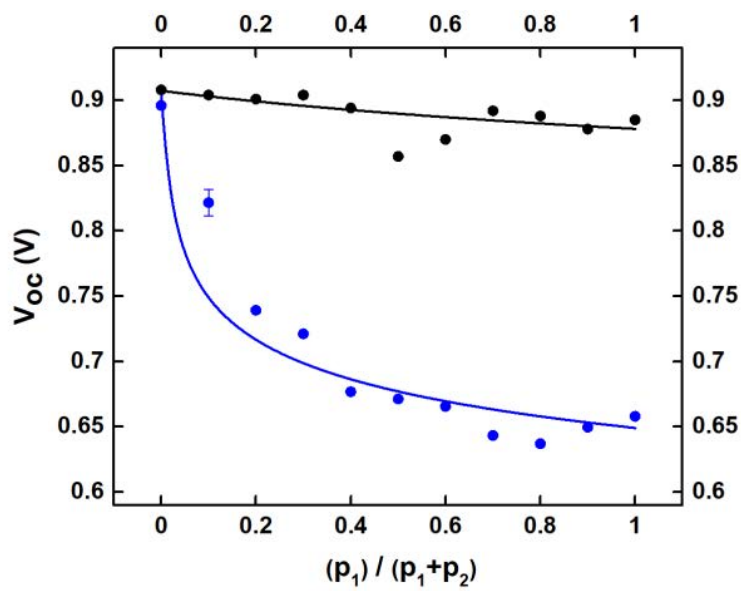

Figure 16. Experimental $V_{\text {oc }}$ vs donor stoichiometry for large (blue symbols) and small (black symbols) HOMO difference reproduced by the state filling model (lines). Adapted from Energy \& Environmental Science, 9, 257-266 (2016) with permission from The Royal Society of Chemistry

Using the same model to evaluate the effect of the opposing current-voltage mechanism, it is predicted that for a constant fill factor, ternary systems do not offer advantages over binary. This is illustrated in Figure 17 where a wide range of donor HOMO levels, i.e. different polymer bandgaps, is evaluated for all compositions; it should be noted that in the above calculation the FF is constant. In this case the favorable compositions are almost entirely binary and they correspond to the highest PCE (cross shape on the right panel of figure 17) hence ternary blends do not enhance the performance of OSCs.
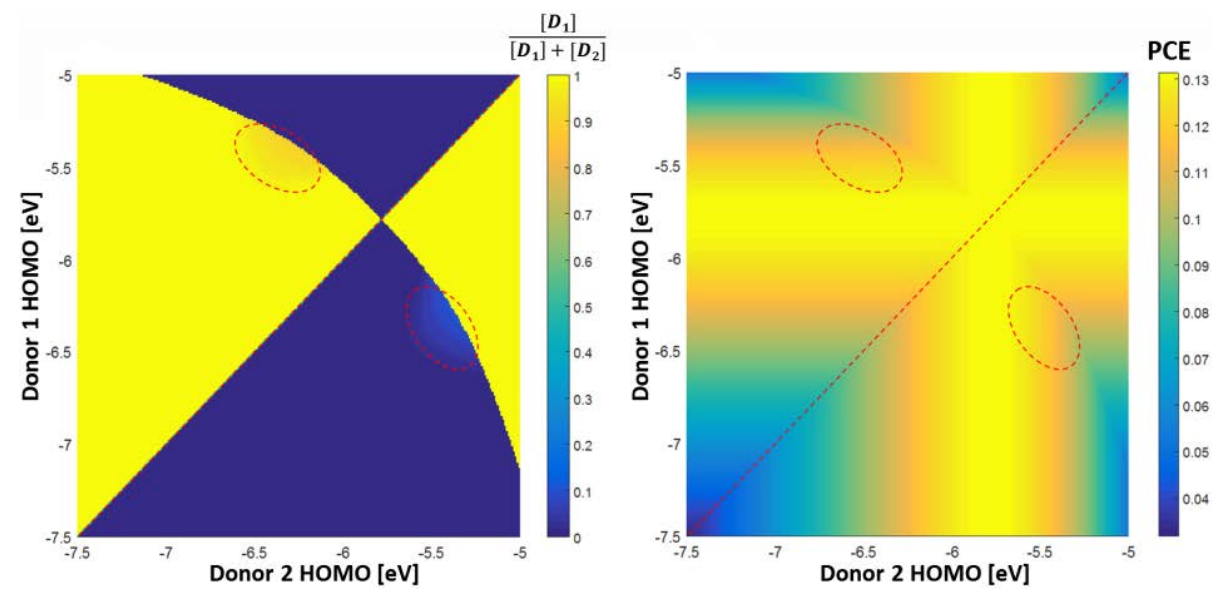

Figure 17. Optimal composition (left) and corresponding PCE (right) for different donor HOMO energy levels calculated for a constant FF. Dashed ellipses indicate ternary composition regions (left) that do not yield the highest overall PCE (right). Reproduced from Energy \& Environmental Science, 9, 257266 (2016) with permission from The Royal Society of Chemistry 
The above finding comes as a surprise since many papers demonstrate ternary compositions that actually improve the PCE of binaries. ${ }^{37,38}$ The explanation lies in the FF, which is often significantly improved for specific ternary blends. If a similar FF variation, with a local maximum at a $50 \% D_{1} D_{2}$ stoichiometry, is included in the state filling model, the best compositions are dominated by ternary blends which correspond to the highest PCE (Figure 18).
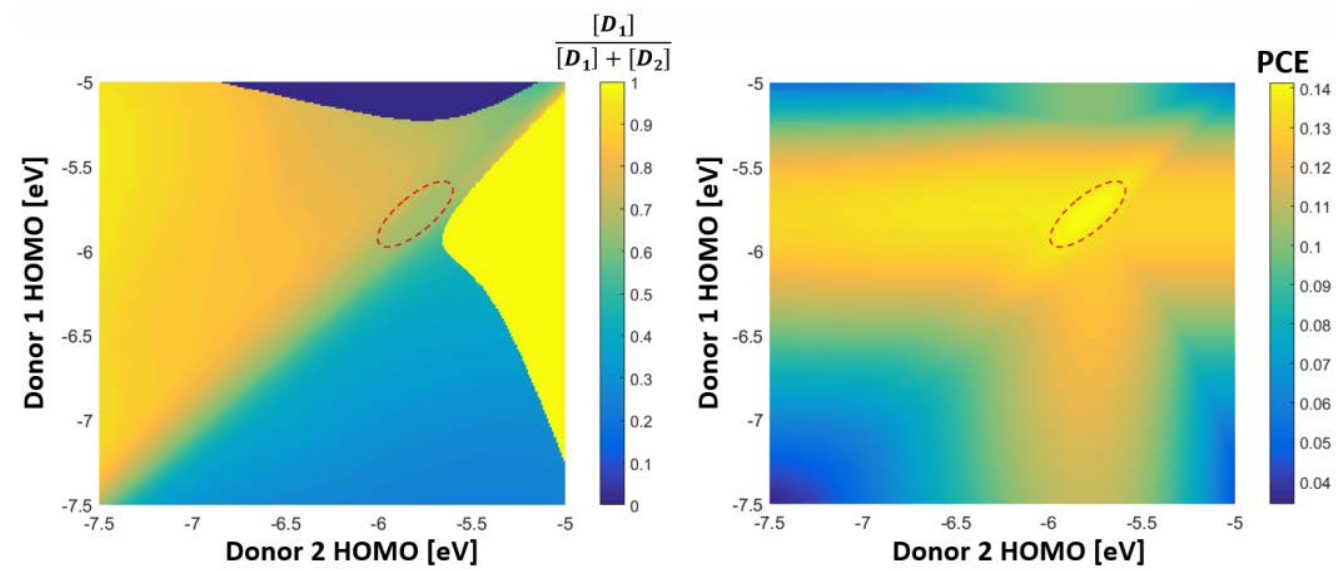

Figure 18. Optimal composition (left) and corresponding PCE (right) for different donor HOMO energy levels calculated for a composition-dependent FF. Dashed ellipses indicate ternary composition regions (left) that result in the highest overall PCE (right). Reproduced from Energy \& Environmental Science, 9, 257-266 (2016) with permission from The Royal Society of Chemistry

\subsection{Donor-Acceptor 1 -Acceptor $2\left(\mathrm{DA}_{1} \mathrm{~A}_{2}\right)$ Ternary BHJ}

It is common to use constant values for the mobility and energetic disorder of a material system, disregarding the stoichiometry. ${ }^{39}$ Following the intriguing finding related with the increased fill factor upon material blending, the mobility and energetic disorder were examined as a function of the blend stoichiometry, where significant variations are expected. ${ }^{40}$ Studying the effect of two different materials on the energetic disorder, a series of ternary $D A_{1} A_{2}$ polymer-fullerenes OPVs was fabricated. These ternary structures have attracted considerably less interest by the research community as fullerene acceptors are poor absorbers and material mixing would not have a significant effect on the photocurrent. The extracted energetic disorders for the HOMO have a local minimum while for the LUMO they are monotonously increasing with acceptor composition, for a ternary TQ1:PC ${ }_{71} B M: I C_{60} B A$ material system (inset of Figure 19). 


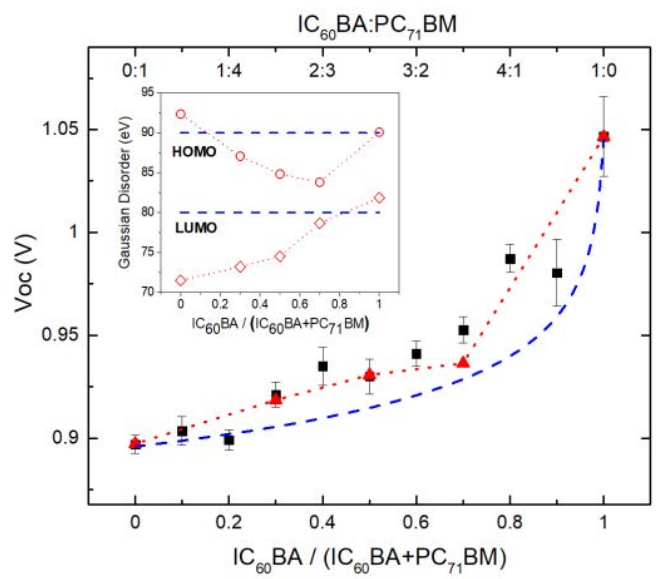

Figure 19. Composition-dependent HOMO,LUMO disorders (inset) reproduce the experimental $\mathrm{V}_{\text {oc }}$ vs acceptor composition data (black symbols) with the equilibrium model (red traces). Reproduced from Applied Materials and Interfaces, 9, 37070-37077 (2017) with permission from the American Chemical Society

Using the extracted variable energetic disorders as input to the state filling equilibrium model, the surprising quasi-linear $V_{o c}$ dependence on the acceptor stoichiometry was reproduced, highlighting the impact of a stoichiometry-dependent energetic disorder. Further analysing the effect of the energetic disorder on the PCE of binary OSCs, a very encouraging $25 \%$ relative improvement can be expected, if upon material blending a small pristine disorder of $60 \mathrm{meV}$ can be maintained (Figure 20).

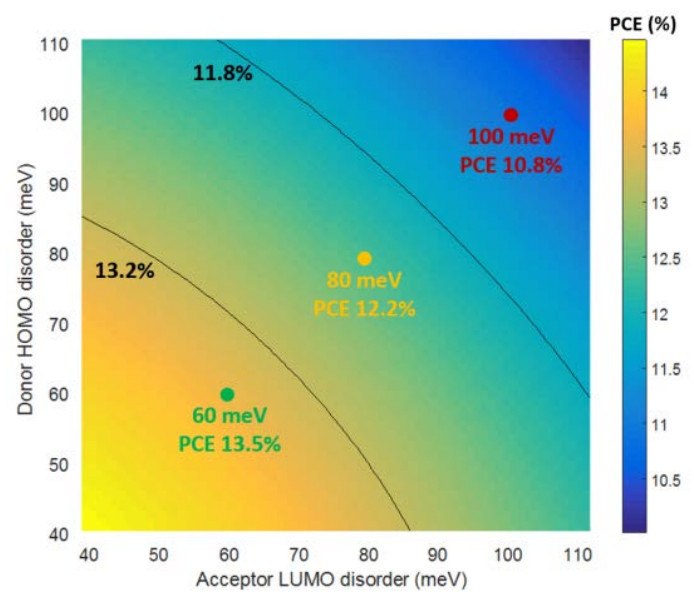

Figure 20. PCE vS HOMO-LUMO energetic disorder of binary OSCs. Maintaining pristine material disorder (60meV) results in a relative increase of the PCE by $25 \%$ (compared to $100 \mathrm{meV}$ ). Adapted from Applied Materials and Interfaces, 9, 37070-37077 (2017) with permission from the American Chemical Society 


\section{Optoelectronic processes of CT states}

Understanding the role of CT states is vital in BHJ OSCs in order to reduce the energy loss paths and increase their performance. Optoelectronic experiments combined with the kMC model shed light on the role of photon energy, energetic disorder and delocalization in the dissociation efficiency of the CT states as well as the origin of CT emission and absorption.

\subsection{Quantum efficiency}

The conversion efficiency of light (incoming photons) to electricity (extracted electrons) is called External Quantum Efficiency (EQE) and is measured as a function of wavelength according to:

$$
E Q E(\lambda)=\frac{\text { electrons }(\lambda)}{\text { photons }(\lambda)}
$$

which is the ratio of extracted electrons to incoming photons per wavelength. Experimentally, EQE is the measured photocurrent for different excitation wavelengths. A monochromator converts a white light source to a single wavelength beam which excites the sample under the application of an external field. A chopper and a lock-in amplifier are usually employed to modulate, demodulate and improve the signal quality. The measured photocurrent vs excitation energy is scaled according to a reference photodetector (usually a silicon photodiode) to provide an absolute EQE value. A more accurate way of scaling the EQE of an OSC is to convolute the EQE spectrum with the AM1.5 spectrum and calibrate the integrated current to the measured $\mathrm{J}_{\mathrm{sc}}$ under $\sim 1$ Sun illumination.

A more advanced technique to measure EQE is called Fourier Transform Photocurrent Spectroscopy (FTPS) and is based on the FTIR (Fourier Transform Infrared Spectroscopy) where the solar cell is used as an external detector. ${ }^{41,42}$ EQE spectra for both experimental methods are shown in Figure 21. 

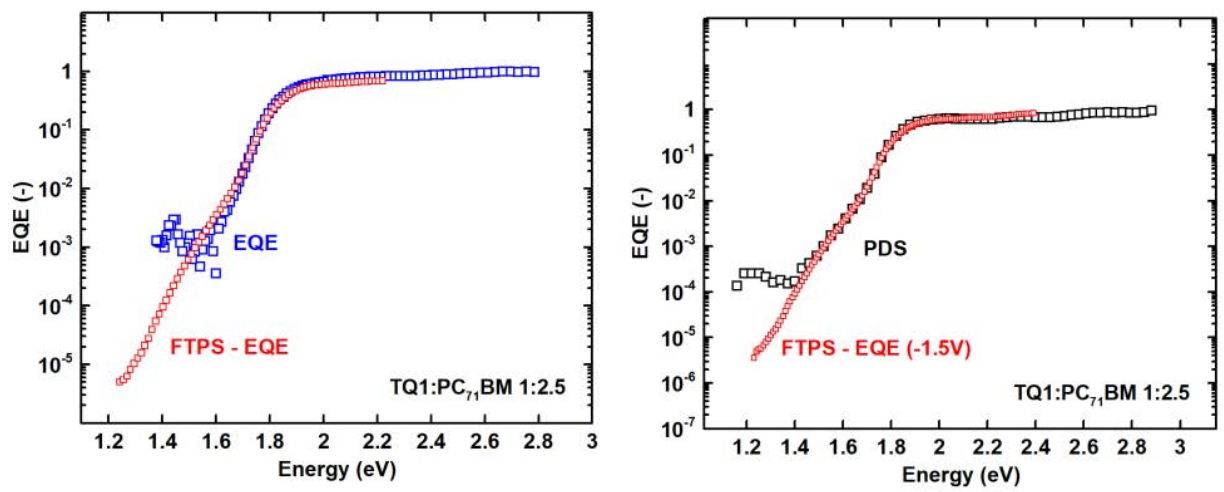

Figure 21. (left) Conventional EQE (blue trace) and FTPS-EQE (red trace) spectra for TQ1:PC ${ }_{71} B M$ measured at OV applied voltage. (right) Absorption from PDS (black trace) and FTPS-EQE spectrum (measured at -1.5V). Adapted with permission.

Another important figure of merit for solar cells is the ability to convert absorbed photons to electrons. The absorption spectrum of the device can be conventionally measured by UV-Vis spectroscopy. A more advanced technique called Photothermal Deflection Spectroscopy (PDS) was used in this thesis, increasing the sensitivity at low photon energies (Figure 21). ${ }^{43}$ In this technique the change of the refractive index of the medium above the film is measured as a result of the heating of the sample upon light absorption. An inert gas on the surface of the sample causes the refraction of the beam for different excitation energies resulting in a measure of the heat and hence an absorption spectrum of the sample. Using the absorption spectrum, the Internal Quantum Efficiency (IQE) can be calculated as

$$
\operatorname{IQE}(\lambda)=\frac{E Q E(\lambda)}{\text { Absorption }(\lambda)}
$$

describing the ratio of extracted electrons to absorbed photons. As shown in Figure 21, the FTPS-EQE spectrum (measured at a reverse bias of $-1.5 \mathrm{~V}$ ) has an identical shape with that of PDS, while it accesses lower excitation energies. It can therefore be used as an absorption spectrum to allow the calculation of the IQE spectrum for excitation energies deep in the tails of the DOS. The absolute IQE value can be determined by optical modeling in which all the individual layers of the device (interlayers, active layer, contacts) are accounted for (parasitic) absorption and reflection according to the Transfer Matrix Method (TMM).$^{44}$ In this way the absorptance of the active layer can be calculated and produce an absolute IQE value using the absolute EQE (equation 24). 
The measured IQE of organic solar cells is surprisingly high (80-90\%), despite the strongly Coulombically bound electron-hole pairs. The role of excitation energy on the efficient charge separation is still under debate; excess photon energy has been claimed to increase the IQE ${ }^{45-47}$, while on the other hand, efficient and energy-independent dissociation yield has been demonstrated over a broad spectrum, i.e. even at low excitation energies. ${ }^{48,49}$ Intuitively, it is expected that the electrons would recombine with the holes when excited deep in the tail of the DOS where the localized sites act as traps and there is no excitation energy available to move and escape. Different proposed mechanisms attempt to explain the surprisingly efficient charge separation, among which are excess phonon energy ${ }^{50}$, the existence of interfacial dipoles ${ }^{51}$ as well as the role of entropy ${ }^{52}$ and energetic disorder. ${ }^{53,54}$ An increasing amount of evidence demonstrates that the electron-hole CT pair delocalizes over an average distance of $3-5 \mathrm{~nm}$, following photoexcitation and exciton creation/diffusion. ${ }^{55-58}$ This physical separation of the CT state significantly reduces the CT binding energy and enables efficient free charge generation. ${ }^{59,60}$ As opposed to inorganic crystalline materials where rapid thermalization, i.e. loss of energy in the continuous bandtype DOS is unavoidable, the intrinsic high energetic disorder of the DOS in organic materials causes charge carriers to thermalize slowly. Hence, electrons and holes can use the available energy to move in the direction of the field and separate, overcoming the Coulomb binding energy. ${ }^{61-63}$

Experimental IQE spectra for two material systems (TQ1:PC $\left.{ }_{71} B M, P C D T B T: P C_{61} B M\right)$ confirm the excitation energy independence at high (up to $2.2 \mathrm{eV}$ ) excitation energies, while at low excitation energies, the dissociation efficiency is reduced/remains independent, respectively, as discussed in paper 5 (Figure 22).

\subsection{Role of CT delocalization}

To understand the photon energy dependence of the experimental IQE, kMC simulations were employed allowing the study of the long-range charge separation, i.e. after all ultrafast processes (not considered in the model) are completed and an electron hole CT pair is created. One of the advantages of kMC is probing very low photon energies, where the reduced excitation energy is comparable to the Coulomb binding energy hence charge separation is critical. It should be mentioned that in these ranges, the extremely low experimental photocurrents (sub-picoAmps) cannot be measured by conventional measurement setups due to low SNR. 
At the beginning of the simulation the excitation energy and the physical e-h separation can be defined, where the latter represents the effect of electron delocalization in the acceptor, following the ultrafast processes of absorption, exciton diffusion and dissociation at the interface. A single electron-hole pair is created and the charge carriers use the excitation, thermal and field energy to move in the disordered energy landscape and either escape to the contacts or recombine. The process is repeated and averaged for many configurations and excitation energies, producing an average IQE spectrum. The simulation results suggest that initial delocalization by at least $4-5 \mathrm{~nm}$ is required to have efficient charge separation at low excitation energies, in agreement with electron delocalization values reported in literature. ${ }^{55-58}$ On the other hand, strongly bound e-h pairs, i.e. pairs with smaller initial separation, will unavoidably recombine at low photon energies, resulting in a quenched and strongly energy-dependent IQE. Hence, the explanation of the

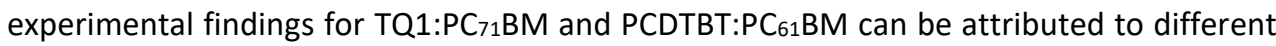
degrees of initial CT pair delocalization lengths (short and long respectively, shown in Figure 22).
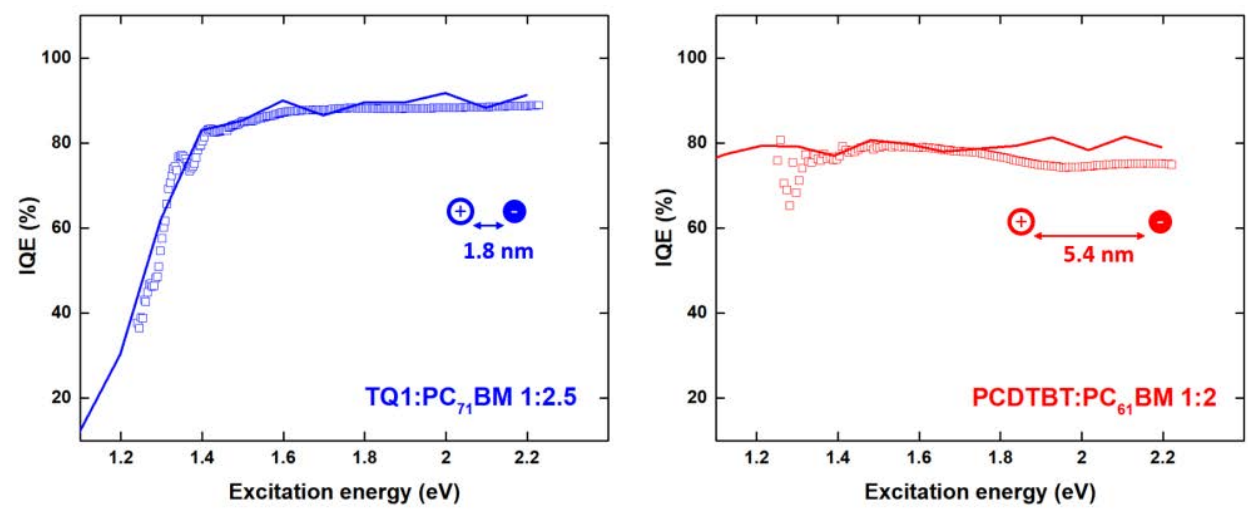

Figure 22. Experimental (symbols) and simulated (lines) IQE spectrum for TQ1:PC $\mathrm{7}_{71} \mathrm{BM}$ (blue traces on the left) and PCDTBT:PC ${ }_{61} \mathrm{BM}$ (red traces on the right). Adapted with permission 


\subsection{Franck-Condon principle}

The spectral shape of the experimental emission and absorption spectra is defined by transitions between the vibronic levels of the ground state and the excited state. ${ }^{64}$ During absorption, the excited electron is promoted from the lowest vibrational level of the ground state $\left(\mathrm{S}_{0}\right)$ to one of the vibrational levels of the excited electronic state $\left(\mathrm{S}_{1}\right)$. Similarly, during emission, an electron undergoes a transition from the lowest vibrational level of the excited state $S_{1}$ to one of the available vibrational levels in $S_{0}$. Both absorption and emission mechanisms are shown in Figure 23

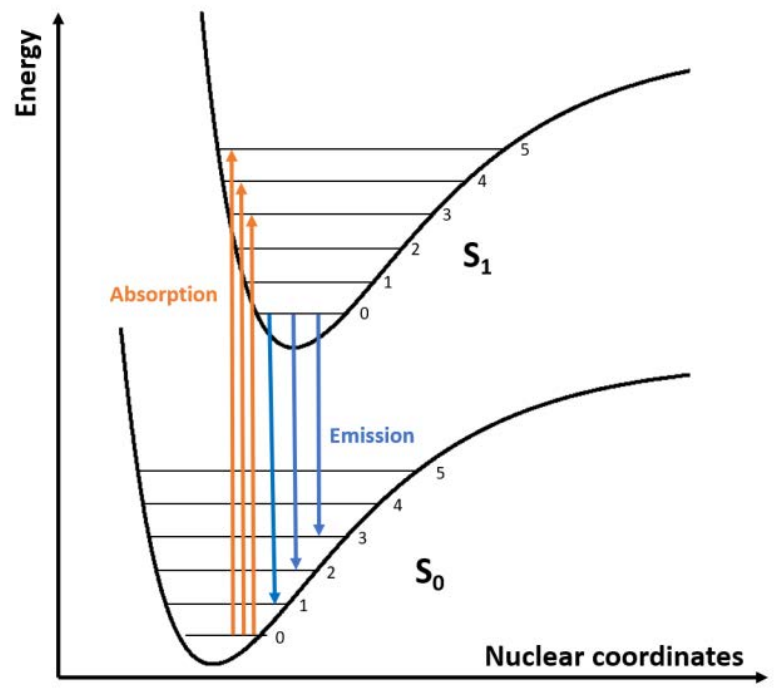

Figure 23. Schematic representation of the Franck Condon principle assuming fast thermalization of the charge carriers and $\mathrm{T}=0 \mathrm{~K}$. Vibrational levels of the electronic states define the energy and shape of emission and absorption.

and are described by the Franck-Condon progression: ${ }^{64}$

$$
I(\hbar \omega) \propto(\hbar \omega)^{3} \times \sum_{m} \frac{S^{m}}{m !} e^{-S} \times \Gamma \times \delta\left(\hbar \omega-\left(\hbar \omega_{0}-m \hbar \omega_{m}\right)\right)
$$

In the above equation, $\mathrm{S}$ is the the Huang-Rhys factor (scaling factor), $\hbar \omega_{0}$ is the central energy of the 0-0 transition, $m$ is the number of contributing vibrational levels (number of peaks), and $\hbar \omega_{m}$ is the energy shift between the peaks (phonon mode). $\Gamma$ is a function accounting for the broadening of the transitions due to the intrinsic energetic disorder and is usually either a Gaussian or a Lorentzian. In this work (papers 4 and 5) the shape of the $\ulcorner$ function was calculated for the CT-EL from KMC simulations using a Gaussian DOS shape 
for the HOMO and LUMO of the donor and acceptor respectively, i.e. the CT-S 0 transition which follows the same principle as $\mathrm{S}_{1}-\mathrm{S}_{0}$ shown in Figure 23. The CT absorption was calculated as the convolution of the two Gaussians. Both methodologies resulted in a Gaussian shape for the $\Gamma$ function and are discussed in chapters 6.5 and 6.6.

\subsection{FLN Spectroscopy}

To obtain the phonon mode and the number of contributing vibrational levels, Fluorescence Line-Narrowing Spectroscopy (FLNS) was employed, which is a highresolution photo-luminescence technique used to measure the optoelectronic spectra of molecular systems. ${ }^{65,66}$ The sample is photo-excited with a certain excitation energy and emits light at a lower energy according to the mechanism shown in Figure 24. Single vibronic transitions are homogeneously thermally broadened $(\sim \mathrm{kT})$ and contribute in total to an inhomogeneous broadening of the absorption/emission spectrum. This is demonstrated in Figure 24 where single vibronic peaks of different magnitude create the total emission spectrum. By reducing the temperature of the sample (down to 20K), the thermal broadening of the vibronic level can be effectively eliminated, concomitantly reducing the inhomogeneous broadening of the total spectrum. A narrow-width laser beam excites the tail of the CT absorption band-edge, i.e. a small subset of the available sites, minimizing the broadening of the total emission spectrum. This technique is used to induce photo-luminescence for a series of material systems used in OSCs (paper 4). Following photo-excitation, the emission energies are detected by a reference photodiode while a chopper and a lock-in amplifier decrease the noise of the detected signal (Figure 24).
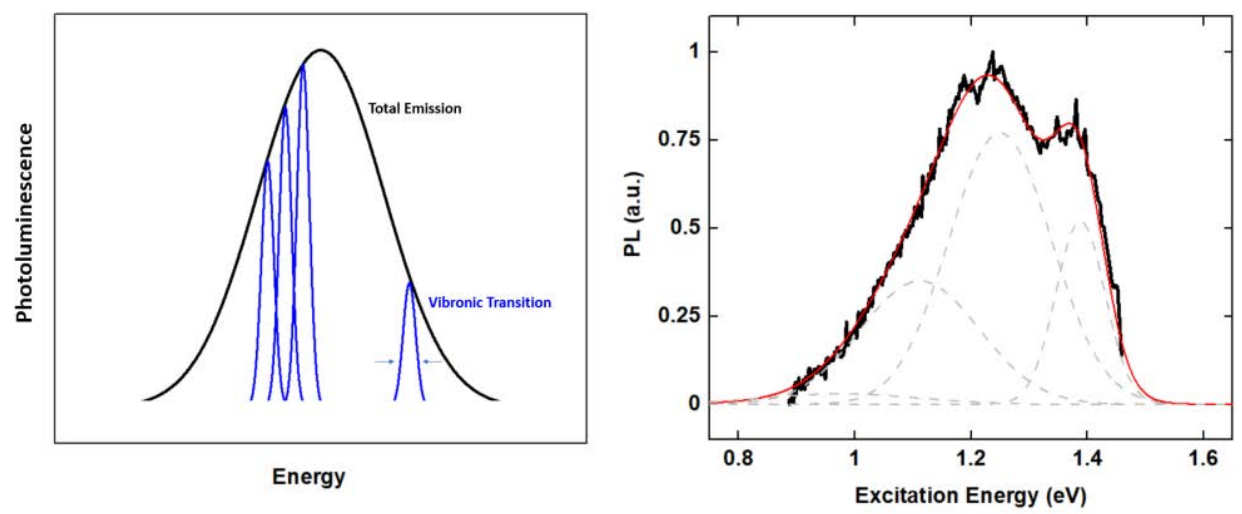

Figure 24. (left) Schematic representation of the total emission spectrum and the single vibronic levels. (right) $\mathrm{FLN}$ photoluminescence (black trace) for TQ1:PC ${ }_{71} \mathrm{BM}$ fitted by a vibronic progression of 4 peaks. 


\subsection{Origin of CT Electroluminescence}

Electroluminescence (EL) is the conversion of electrical power to light and can be generated when a solar cell is operated as a light emitting diode. A field is applied to the device via the contacts, injecting electrons (holes) in the acceptor LUMO (donor HOMO). The thermalized charge carriers are transported in the device, until they meet and recombine under emission of light. This light is detected by a reference photodiode at different wavelengths resulting in the CT-EL spectrum (Figure 25). Understanding the origin of the CT-EL is crucial in analyzing the energetics of the CT states and the relationship between emission and absorption, identifying loss mechanisms and potential routes to efficiency enhancement, as introduced in chapter 3.2.2.

Using the $\mathrm{kMC}$ model, electrons and holes from the contacts are monitored in time, energy and space dimensions from the injection to the recombination event. The 0-0 transition between $\mathrm{S}_{0}$ and $\mathrm{CT}$ is simulated accounting for the Gaussian energetic disorder, attempt frequency and lattice constant (Figure 25).
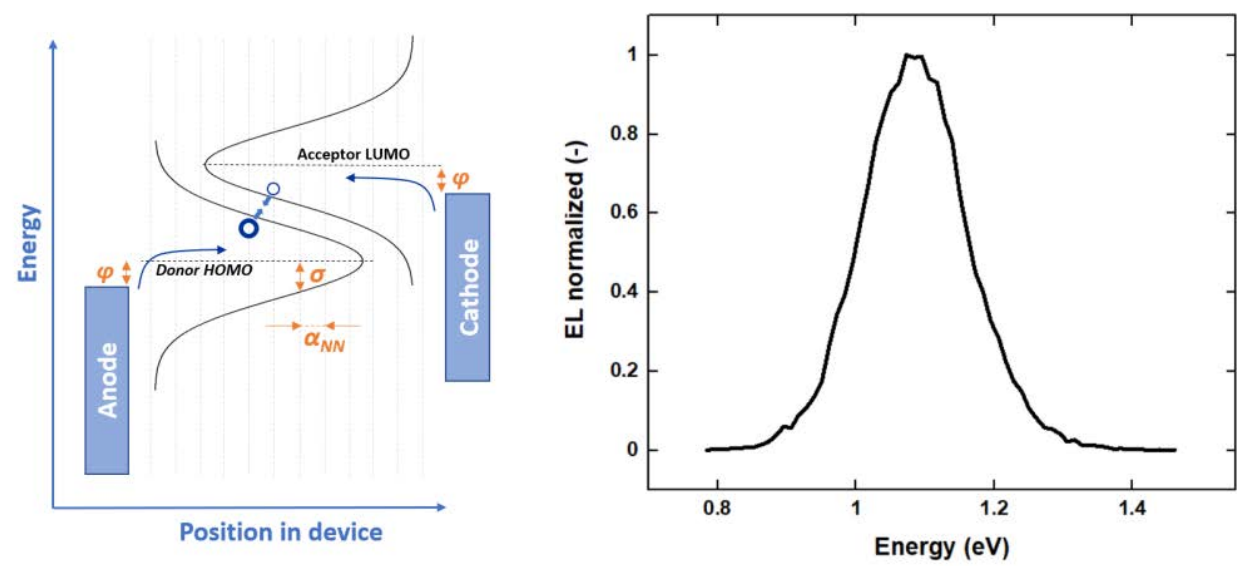

Figure 25. (left) Schematic representation of a kMC electroluminescence simulation. Charge carriers are injected from the contacts, travel in the device and recombine. Energetic disorder, lattice constant and injection barriers are shown as $\sigma, \alpha_{N N}$ and $\varphi$. (right) CT-EL spectrum from kinetic Monte Carlo simulations.

The resulting spectrum width, using energetic disorders independently measured from SCLC experiments, is surprisingly only a fraction of the experimental CT-EL spectrum. Using the Franck-Condon principle, the total peak shape is perfectly reproduced with a progression of the simulated KMC 0-0 peak for the number of vibrational peaks found experimentally for numerous material systems (paper 4). Interestingly and in contrast to 
conventional wisdom, the energy levels from which the EL CT originates are only a nonthermalized subset of all the available states in the effective DOS.

\subsection{Fitting the entire optical spectrum}

A common method of describing the CT-EL and CT-Absorption spectrum is based on the reciprocity of their origin, i.e. the fact the same energy states are thought to participate in both processes. ${ }^{23}$ Identical Gaussians are fitted to both experimental data according to this method, shifted by the reorganization energy and their crossing point is taken as the CT energy, as stated earlier. ${ }^{23}$ This method could reasonably well reproduce the high (low) energy part of the CT-EL (Absorption) spectra as well as the entire range of emission and absorption spectra for two materials systems ( paper 5) as shown in Figure 26.
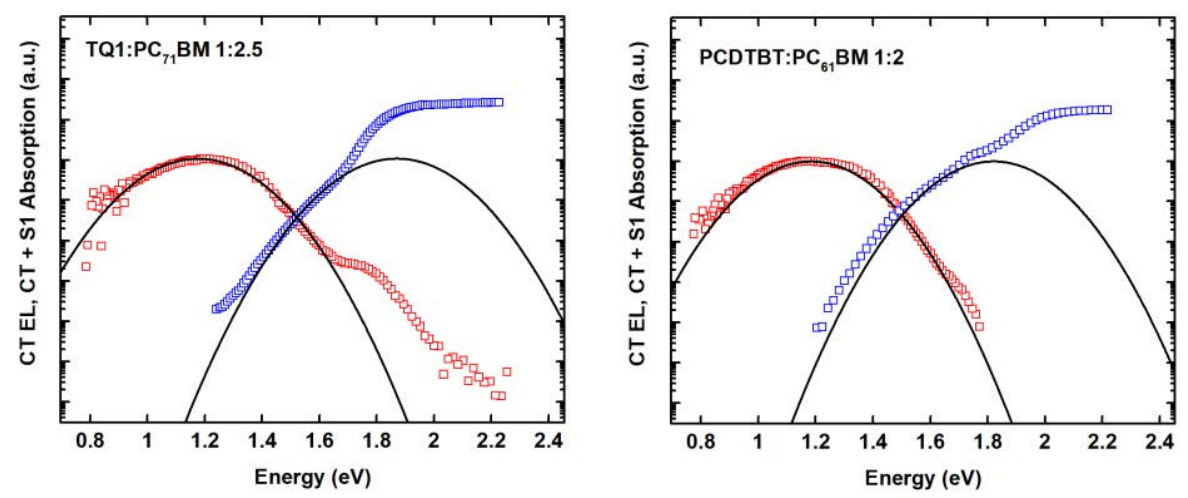

Figure 26. $\mathrm{CT}$ electroluminescence and absorption fitted with parabolas according to Ref. 23 for TQ1:PC ${ }_{71} \mathrm{BM}$ (left) and PCDTBT:PC ${ }_{61} \mathrm{BM}$ (right). Reproduced with permission.

Alternatively, as part of this thesis, the entire CT-EL / Absorption optical spectrum was reproduced independently using the same material parameters, i.e. Gaussian disorder for the HOMO and LUMO as well as the absolute position of the HOMO, LUMO levels. ${ }^{26,67,68}$ CT-Absorption was calculated as the convolution of two Gaussian distributions (HOMO and LUMO DOS), i.e. $g_{\text {holes }} * g_{\text {electrons }}$. This is in strong contrast to the origin of the CT-EL as discussed earlier, i.e. no direct reciprocity is assumed between the two spectra. The results of the fits to the CT EL and CT Absorption are in very good agreement with the experiments as shown in Figure 27. The extracted energy levels and disorders were used for the kMC IQE simulations of Figure 22. 

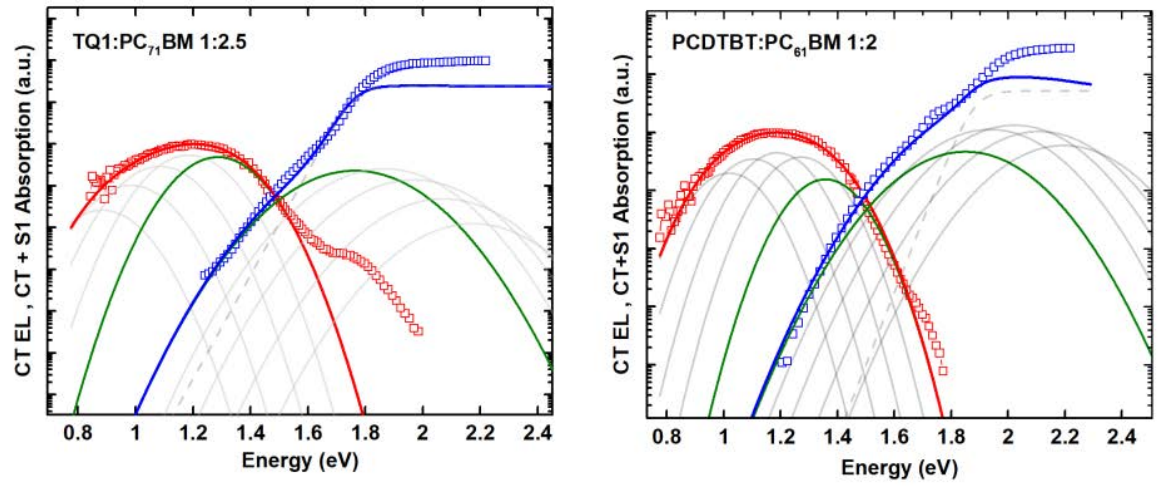

Figure 27. Experimental (symbols) and simulated (lines) IQE spectrum for TQ1:PC ${ }_{71} \mathrm{BM}$ (blue traces on the left) and PCDTBT:PC ${ }_{61} \mathrm{BM}$ (red traces on the right). Reproduced with permission. 


\subsection{Transient photocurrent experiment limits}

Charge carrier extraction of photo-created electron-hole pairs occurs on time scales that extend from sub-picoseconds to microseconds in OSCs. Due to the intrinsic energetic disorder, gradual thermalization of the charge carriers results in dispersive mobilities experimentally observed at early timescales $(<\mu s)$ that needs to be accounted for in simulations. ${ }^{26,69,70}$ Slower processes can be satisfyingly described by equilibrium charge transport models, using a constant mobility.

The transient photocurrent (TPC) experiment is easy to perform and a direct way to study the time dependence of charge transport and extraction in OSCS on timescales larger than the RC time. A light source illuminates the device which is connected to an oscilloscope resolving the transient signals. The light source is stepped and the charge carriers' transient response to the change is probed as a function of time. The measurement setup (oscilloscope bandwidth, RC from measurement cables, connections and the sample itself) set the time resolution of the experiment (commonly in the range of microseconds) (Figure 28).

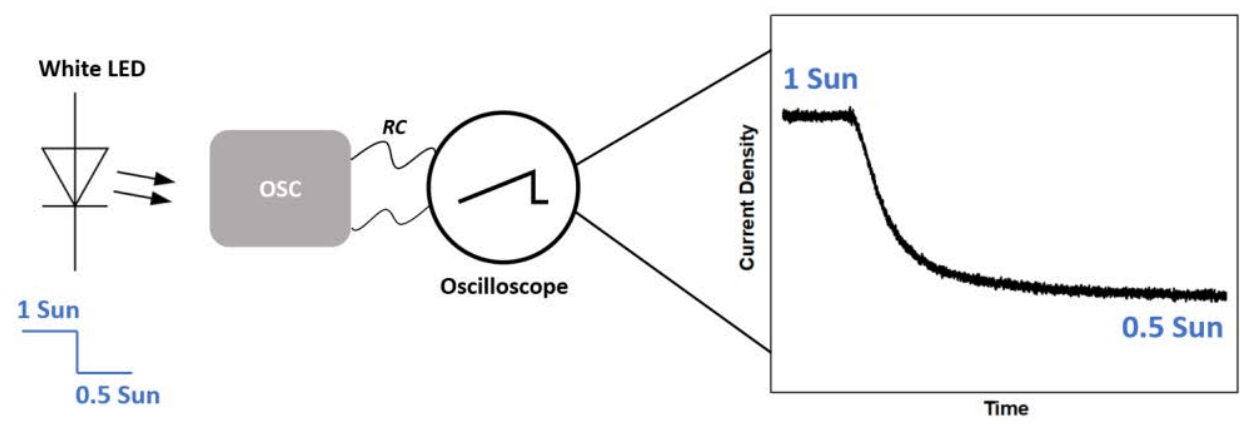

Figure 28. Schematic representation of the TPC experiment and the resulting current density vs time data for a 1 to 0.5 Sun illumination intensity step.

A significantly faster technique is Time Resolved Electric Field Induced Second Harmonic (TREFISH) resolving picosecond transient signals. This technique is used to probe thermalization and conductivity dispersion in OSCs. ${ }^{71,72}$ TREFISH relies on the second harmonic generation effect induced by the presence of a field, i.e. the interaction of two photons having the same frequency with a suitable material that creates a new photon of double frequency. Changes in the field which is partially screened by the movement of 
photo-created charge carriers in the device, can be probed optically and are related to extracted charge carriers. Combined with TPC measurements, TREFISH can probe timescales from $100 \mathrm{fs}$ to $10 \mu \mathrm{s}$.

Based on TPC measurements with $\sim \mu$ s resolution described by DD equations with a constant mobility, it was claimed that ultrafast $\sim$ ns non-equilibrium charge transport is not relevant for operating OSCs. ${ }^{73}$ The fact that TPC is 'blind' to all events faster than $\sim \mu \mathrm{s}$, is the reason why other ultrafast probing experiments as e.g. TREFISH are preferably used. The TPC experimental transients was successfully described with DD equations using a constant mobility, however this is only relevant for the slow/trapped thermalized charge carriers that the specific experiment resolves. In paper 7, the TPC experiment is repeated and described successfully with $\mathrm{kMC}$ simulations using energetic disorder parameters from earlier work, in which TREFISH experiments are also successfully reproduced. ${ }^{26}$ Analyzing the extraction time distribution of the charge carriers, it is shown that the TPC experiment probes only a subset of the total charge carriers, as most electrons and a fraction of the holes are extracted at much earlier time scales than the experiment resolves (Figure 29). The slow relaxation and dispersive motion is a result of the intrinsic energetic disorder which prevents fast thermalization, i.e. energy loss of the charge carriers. However, this needs to be considered as a non-worst-case scenario and not a design rule as increased energetic disorder generally limits the performance of OSCs.
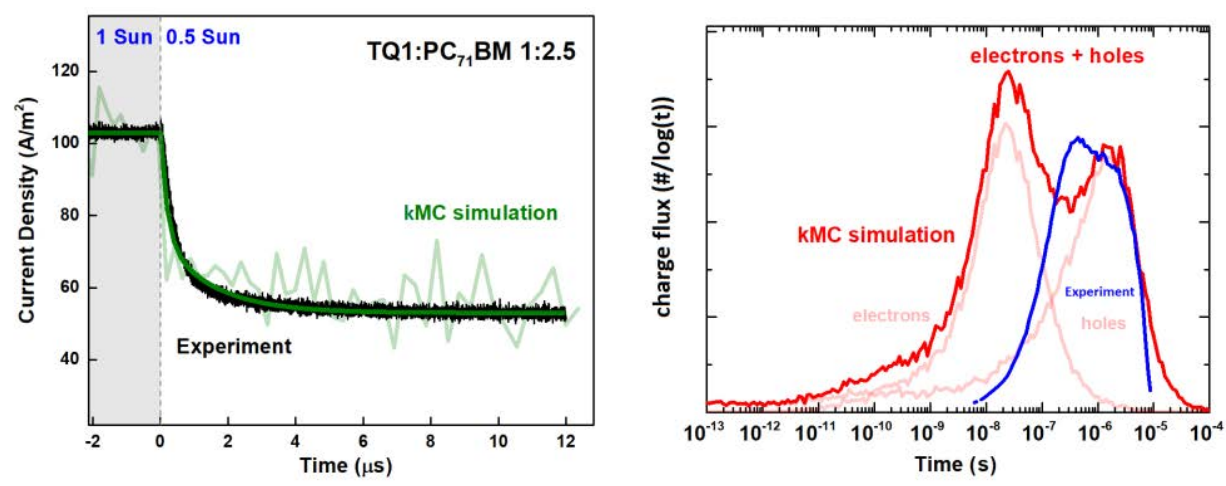

Figure 29. (left) Transient photocurrent experiment (black trace) and kMC simulation (green trace). (right) Histogram of simulated electrons and holes (red traces) and TPC experiment (blue trace) for TQ1:PC ${ }_{71} B M$. RC time is $0.3 \mu \mathrm{s}$. Adapted from Advanced Energy Materials (2018) with permission from John Wiley and Sons. 


\section{Conclusion}

Concluding this work, we have shown that the intrinsic energetic disorder in organic semiconductors plays a key role in the optoelectronic properties and the performance of OSCs. Parametrizing the charge carrier mobility according to the extended Gaussian disorder model, successfully reproduces steady-state experiments with equilibrium models. Using the same formalism (eGDM) to describe the energetics and the thermally activated hopping transport, stochastic KMC models reproduce accurately non-equilibrium experiments. Steady-state SCLC experiments can be analyzed automatically to extract charge carrier mobilities and energetic disorder according to different analytical models, via the use of an automated fitting tool.

Energetic disorder is found to vary with the stoichiometry of the blend in binary OSCs where the pristine disorder can be either reduced, increased or even maintained in presence of another material. In ternary OSCs composed of two acceptors, the energetic disorder can have a (semi-) monotonous dependence on the acceptor stoichiometry and is found to directly determine the surprising linearity of $V_{o c}$, when accounted for in an equilibrium joint Gaussian DOS state-filling model. Maintaining the low pristine energetic disorder can considerably improve the performance of OSCs. In ternary blends with two donors $\mathrm{V}_{\mathrm{oc}}$ is surprisingly not pinned to the shallowest HOMO while it varies with donor stoichiometry allowing for it to be controlled/designed. Introducing a second donor to increase the absorption spectral coverage and therefore the current density (while concomitantly reducing the voltage), is shown not to ultimately increase the PCE unless the FF is enhanced upon blending.

The energetics of the CT states probed by CT-EL and CT-absorption is reproduced with a common set of energetic disorder parameters and energy levels. Using the Franck-Condon principle the CT-EL is reproduced by a vibronic progression of the KMC simulated $\mathrm{S}_{0}-\mathrm{S}_{1}$ transition which originates only from a subset of the total DOS. Using the same HOMOLUMO energetic disorder and levels, the CT absorption is calculated as the convolution of the Gaussian HOMO and LUMO DOS accurately reproducing the experimental data. Using the above material parameters, the surprisingly efficient and photon-energy independent charge separation experimentally observed in organic solar cells, is reproduced with kMC simulations, where the delocalization of the CT electron-hole pair plays a key role. Strongly (weakly) bound e-h pairs delocalized over a small (large) distance will result in a decreasing IQE at low excitation energies. An initial electron-hole separation of 4-5nm is necessary for efficient dissociation at low excitation energies. 
Claiming that ultrafast non-equilibrium processes are not relevant for operating OSCS based on a TPC experiment with limited temporal resolution $(\sim \mu s)$ was shown to be unjustified. The TPC experiment was repeated and successfully reproduced by kMC simulations with disorder parameters used to describe ultra-fast ( ps) TREFISH transient data. The extraction time distributions indicate fast extraction of electrons (undetectable by the experiment) followed by slower extraction of (trapped) holes. 


\section{Bibliography}

1. Shockley, W. \& Queisser, H. J. Detailed Balance Limit of Efficiency of pn Junction Solar Cells Detailed Balance Limit of Efficiency of p-n Junction Solar Cells. J Appl Phys Addit. Inf. J Appl Phys J. Homepage 32, (1961).

2. Krebs, F. C., Tromholt, T. \& Jørgensen, M. Upscaling of polymer solar cell fabrication using full roll-to-roll processing. Nanoscale 2, 873 (2010).

3. Zhao, W. et al. Fullerene-Free Polymer Solar Cells with over $11 \%$ Efficiency and Excellent Thermal Stability. Adv. Mater. 28, 4734-4739 (2016).

4. Li, M. et al. Solution-processed organic tandem solar cells with power conversion efficiencies \textgreater12\%. Nat. Photonics 11, 85-90 (2017).

5. Søndergaard, R., Hösel, M., Angmo, D., Larsen-Olsen, T. T. \& Krebs, F. C. Roll-to-roll fabrication of polymer solar cells. Mater. Today 15, 36-49 (2012).

6. Blom, P. W. M., de Jong, M. J. M. \& Liedenbaum, C. T. H. F. Device physics of polymer light-emitting diodes. Polym. Adv. Technol. 9, 390-401 (1998).

7. Bharti, D., Varun, I. \& Tiwari, S. P. Performance enhancement in TIPS-pentacene:PS blend organic field effect transistors by solvent vapor annealing. in 2016 74th Annual Device Research Conference (DRC) 1-2 (IEEE, 2016).

8. Kuik, M. et al. 25th Anniversary Article: Charge Transport and Recombination in Polymer Light-Emitting Diodes. Adv. Mater. 26, 512-531 (2014).

9. Tanase, C., Meijer, E. J., Blom, P. W. M. \& De Leeuw, D. M. Unification of the Hole Transport in Polymeric Field-Effect Transistors and Light-Emitting Diodes. Phys Rev Lett, 91, 216601 (2009).

10. Pasveer, W. F. et al. Unified Description of Charge-Carrier Mobilities in Disordered Semiconducting Polymers. Phys Rev Lett, 94, 206601 (2005).

11. Leighton, P. A. Electronic Processes in Ionic Crystals (Mott, N. F.; Gurney, R. W.). J. Chem. Educ. 18, 249 (1941).

12. Zuo, G. et al. Molecular Doping and Trap Filling in Organic Semiconductor Host-Guest Systems. J. Phys. Chem. C, 121, 7767 (2017).

13. Felekidis, N., Melianas, A. \& Kemerink, M. Design Rule for Improved Open-Circuit Voltage in Binary and Ternary Organic Solar Cells. ACS Appl. Mater. Interfaces $\mathbf{9}$, (2017).

14. Murgatroyd, P. N. Theory of space-charge-limited current enhanced by Frenkel effect. J. Phys. Appl. Phys. 3, 308 (1970).

15. Heiber, M. C. et al. Impact of Tortuosity on Charge-Carrier Transport in Organic Bulk Heterojunction Blends. Phys. Rev. Appl. 8, 054043 (2017).

16. Bässler, H. Charge Transport in Disordered Organic Photoconductors a Monte Carlo Simulation Study. Phys. Status Solidi B 175, 15-56 (1993).

17. Bouhassoune, M., van Mensfoort, S. L. M., Bobbert, P. A. \& Coehoorn, R. Carrierdensity and field-dependent charge-carrier mobility in organic semiconductors with correlated Gaussian disorder. Org. Electron. 10, 437-445 (2009). 
18. Marianer, S. \& Shklovskii, B. I. Effective temperature of hopping electrons in a strong electric field. Phys. Rev. B 46, (1992).

19. Cottaar, J., Koster, L. J. A., Coehoorn, R. \& Bobbert, P. A. Scaling Theory for Percolative Charge Transport in Disordered Molecular Semiconductors. Phys. Rev. Lett. 107, 136601 (2011).

20. Nenashev, A. V. et al. Advanced percolation solution for hopping conductivity. Phys. Rev. B 87, 235204 (2013).

21.Craciun, N. I., Wildeman, J. \& Blom, P. W. M. Universal Arrhenius Temperature Activated Charge Transport in Diodes from Disordered Organic Semiconductors, 100, 056601, (2008).

22. M.Kemerink. FitSCLC. (https://github.com/mkemerink/FitSCLC, 2018).

23. Vandewal, K., Tvingstedt, K., Gadisa, A., Inganäs, O. \& Manca, J. V. Relating the opencircuit voltage to interface molecular properties of donor:acceptor bulk heterojunction solar cells. Phys. Rev. B 81, 125204 (2010).

24. Felekidis, N. OPV Weight Calculator. Available from: (https://play.google.com/store/apps/details?id=com.OPV.NF.OPV_WeightCalculator\& hl=en_GB) (2017).

25.van Mensfoort, S. L. M. \& Coehoorn, R. Effect of Gaussian disorder on the voltage dependence of the current density in sandwich-type devices based on organic semiconductors. Phys. Rev. B 78, 085207 (2008).

26. Melianas, A. et al. Dispersion-Dominated Photocurrent in Polymer:Fullerene Solar Cells. Adv. Funct. Mater. 24, 4507-4514 (2014).

27. Baranovskii, S. D. Theoretical description of charge transport in disordered organic semiconductors. Phys. Status Solidi B 251, 487-525 (2014).

28. Miller, A. \& Abrahams, E. Impurity Conduction at Low Concentrations. Phys. Rev. 120, 745-755 (1960).

29. Noolandi, J. Equivalence of multiple-trapping model and time-dependent random walk. Phys. Rev. B 16, 4474-4479 (1977).

30. Khlyabich, P. P., Rudenko, A. E., Thompson, B. C. \& Loo, Y.-L. Structural Origins for Tunable Open-Circuit Voltage in Ternary-Blend Organic Solar Cells. Adv. Funct. Mater. 25, 5557-5563 (2015).

31. Khlyabich, P. P., Burkhart, B. \& Thompson, B. C. Compositional Dependence of the Open-Circuit Voltage in Ternary Blend Bulk Heterojunction Solar Cells Based on Two Donor Polymers. J. Am. Chem. Soc. 134, 9074-9077 (2012).

32.Zhang, Y. et al. Synergistic Effect of Polymer and Small Molecules for HighPerformance Ternary Organic Solar Cells. Adv. Mater. 27, 1071-1076 (2015).

33. Yang, L., Zhou, H., Price, S. C. \& You, W. Parallel-like Bulk Heterojunction Polymer Solar Cells. J. Am. Chem. Soc. 134, 5432-5435 (2012).

34.Savoie, B. M., Dunaisky, S., Marks, T. J. \& Ratner, M. A. The Scope and Limitations of Ternary Blend Organic Photovoltaics. Adv. Energy Mater. 5, 1400891 (2015).

35. Street, R. A., Davies, D., Khlyabich, P. P., Burkhart, B. \& Thompson, B. C. Origin of the Tunable Open-Circuit Voltage in Ternary Blend Bulk Heterojunction Organic Solar Cells. J. Am. Chem. Soc. 135, 986-989 (2013). 
36. Wang, Z. et al. From Alloy-Like to Cascade Blended Structure: Designing HighPerformance All-Small-Molecule Ternary Solar Cells. J. Am. Chem. Soc. 140, 1549-1556 (2018).

37.Um, H. A., Lee, D. H., Park, G. E., Cho, M. J. \& Choi, D. H. Ternary polymer solar cell based on two donors and one acceptor for improving morphology and power conversion efficiency, 220, 362-368 (2016).

38.Zhang, J. et al. Conjugated Polymer-Small Molecule Alloy Leads to High Efficient Ternary Organic Solar Cells. J. Am. Chem. Soc. 137, 8176-8183 (2015).

39.Scharber, M. C. On the Efficiency Limit of Conjugated Polymer:Fullerene-Based Bulk Heterojunction Solar Cells. Adv. Mater. 28, 1994-2001 (2016).

40. Mihailetchi. Device physics of organic bulk heterojunction solar cells. (2017).

41.Vanecek, M. \& Poruba, A. Fourier-transform photocurrent spectroscopy of microcrystalline silicon for solar cells. Appl. Phys. Lett. 80, 719-721 (2002).

42. Vandewal, K. et al. Fourier-Transform Photocurrent Spectroscopy for a fast and highly sensitive spectral characterization of organic and hybrid solar cells. Thin Solid Films 516, 7135-7138 (2008).

43. Vandewal, K., Goris, L., Haenen, K., Geerts, Y. \& Manca, J. V. Highly sensitive spectroscopic characterization of inorganic and organic heterojunctions for solar cells. Eur. Phys. J. Appl. Phys. 36, 281-283 (2006).

44. Burkhard, G. F., Hoke, E. T. \& McGehee, M. D. Accounting for Interference, Scattering, and Electrode Absorption to Make Accurate Internal Quantum Efficiency Measurements in Organic and Other Thin Solar Cells. Adv. Mater. 22, 3293-3297 (2010).

45. Hahn, T. et al. Does Excess Energy Assist Photogeneration in an Organic Low-Bandgap Solar Cell? Adv. Funct. Mater. 25, 1287-1295 (2015).

46. Dimitrov, S. D. et al. On the Energetic Dependence of Charge Separation in Low-BandGap Polymer/Fullerene Blends. J. Am. Chem. Soc. 134, 18189-18192 (2012).

47.Grancini, G. et al. Hot exciton dissociation in polymer solar cells. Nat. Mater. 12, 29-33 (2012).

48. Gautam, B. R. et al. Charge Photogeneration in Organic Photovoltaics: Role of Hot versus Cold Charge-Transfer Excitons. Adv. Energy Mater. 6, 1301032 (2016).

49. Vandewal, K. et al. Efficient charge generation by relaxed charge-transfer states at organic interfaces. Nat. Mater. 13, 63-68 (2013).

50. Arkhipov, V. I., Emelianova, E. V. \& Bässler, H. Hot Exciton Dissociation in a Conjugated Polymer. Phys. Rev. Lett. 82, 1321-1324 (1999).

51.Gregg, B. A. Entropy of Charge Separation in Organic Photovoltaic Cells: The Benefit of Higher Dimensionality. J. Phys. Chem. Lett. 2, 3013-3015 (2011).

52. Monahan, N. R., Williams, K. W., Kumar, B., Nuckolls, C. \& Zhu, X.-Y. Direct Observation of Entropy-Driven Electron-Hole Pair Separation at an Organic Semiconductor Interface. Phys. Rev. Lett. 114, 247003 (2015).

53. van Eersel, H., Janssen, R. A. J. \& Kemerink, M. Mechanism for Efficient Photoinduced Charge Separation at Disordered Organic Heterointerfaces. Adv. Funct. Mater. 22, 2700-2708 (2012). 
54. Albrecht, U. \& Bässler, H. Yield of geminate pair dissociation in an energetically random hopping system. Chem. Phys. Lett. 235, 389-393 (1995).

55. Gelinas, S. et al. Ultrafast Long-Range Charge Separation in Organic Semiconductor Photovoltaic Diodes, 6170, 512 (2014).

56. Barker, A. J., Chen, K. \& Hodgkiss, J. M. Distance Distributions of Photogenerated Charge Pairs in Organic Photovoltaic Cells. J. Am. Chem. Soc. 136, 12018-12026 (2014).

57. Chen, K., Barker, A. J., Reish, M. E., Gordon, K. C. \& Hodgkiss, J. M. Broadband Ultrafast Photoluminescence Spectroscopy Resolves Charge Photogeneration via Delocalized Hot Excitons in Polymer:Fullerene Photovoltaic Blends. J. Am. Chem. Soc. 135, 1850218512 (2013).

58. Bernardo, B. et al. Delocalization and dielectric screening of charge transfer states in organic photovoltaic cells. Nat. Commun. 5, 3245 (2014).

59. Caruso, D. \& Troisi, A. Long-range exciton dissociation in organic solar cells. PNAS 109, 13498-502 (2012).

60. Nenashev, A. V. et al. Theory of exciton dissociation at the interface between a conjugated polymer and an electron acceptor. Phys. Rev. B 84, 035210 (2011).

61. Borsenberger, P. M., Richert, R. \& Bässler, H. Dispersive and nondispersive charge transport in a molecularly doped polymer with superimposed energetic and positional disorder. Phys. Rev. B 47, 4289-4295 (1993).

62. Coehoorn, R., Zhang, L., Bobbert, P. A. \& van Eersel, H. Effect of polaron diffusion on exciton-polaron quenching in disordered organic semiconductors. Phys. Rev. B 95, 134202 (2017).

63. Etzold, F. et al. Ultrafast Exciton Dissociation Followed by Nongeminate Charge Recombination in PCDTBT:PCBM Photovoltaic Blends. J. Am. Chem. Soc. 133, 94699479 (2011).

64. Köhler, A. \& Bässler, H. Electronic Processes in Organic Semiconductors, Wiley-VCH Verlag GmbH \& Co. KGaA, (2015).

65. Fidy, J., Laberge, M., Kaposi, A. D. \& Vanderkooi, J. M. Fluorescence line narrowing applied to the study of proteins. Biochim. Biophys. Acta BBA - Protein Struct. Mol. Enzymol. 1386, 331-351 (1998).

66. Jankowiak, R. \& Small, G. J. Fluorescence line-narrowing spectroscopy in the study of chemical carcinogenesis. Anal. Chem. 61, 1023A-1024A, 1026A-1029A, 1031A-103 (1989).

67. Melianas, A. et al. Photo-generated carriers lose energy during extraction from polymer-fullerene solar cells. Nat. Commun. 6, 8778 (2015).

68. Felekidis, N., Melianas, A. \& Kemerink, M. Nonequilibrium drift-diffusion model for organic semiconductor devices. Phys. Rev. B 94, 035205 (2016).

69. Vithanage, D. A. et al. Visualizing charge separation in bulk heterojunction organic solar cells. Nat. Commun. 4, 2334 (2013).

70. Howard, I. A., Etzold, F., Laquai, F. \& Kemerink, M. Nonequilibrium Charge Dynamics in Organic Solar Cells. Adv. Energy Mater. 4, 1301743 (2014). 
71. Devižis, A., Serbenta, A., Meerholz, K., Hertel, D. \& Gulbinas, V. Ultrafast Dynamics of Carrier Mobility in a Conjugated Polymer Probed at Molecular and Microscopic Length Scales. Phys. Rev. Lett. 103, 027404 (2009).

72. Melianas, A. Non-Equilibrium Charge Motion in Organic Solar Cells. (Linköping University Electronic Press, 2017).

73. Le Corre, V. M., Chatri, A. R., Doumon, N. Y. \& Koster, L. J. A. Charge Carrier Extraction in Organic Solar Cells Governed by Steady-State Mobilities. Adv. Energy Mater. 7, 1701138 (2017). 


\section{Material abbreviations and chemical structure}

\section{TQ1}

poly[[2,3-bis(3-octyloxyphenyl)-5,8-quinoxalinediyl]-2,5-thiophenediyl]

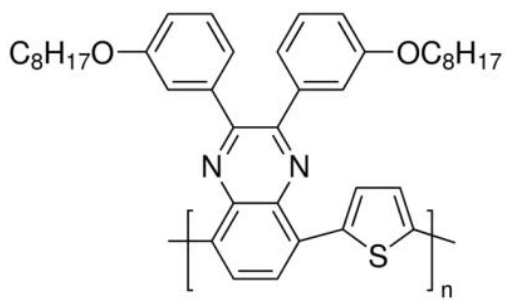

\section{TQm6}

Poly[2,3-bis-(3-hexyloxyphenyl)quinoxaline-5,8-diyl-alt-thiophene-2,5-diyl]

\section{TQm12}

Poly[2,3-bis-(3-dodecyloxyphenyl)quinoxaline-5,8-diyl-alt-thiophene-2,5-diyl]

\section{TQp6}

Poly[2,3-bis-(4-hexyloxyphenyl)quinoxaline-5,8-diyl-alt-thiophene-2,5-diyl]
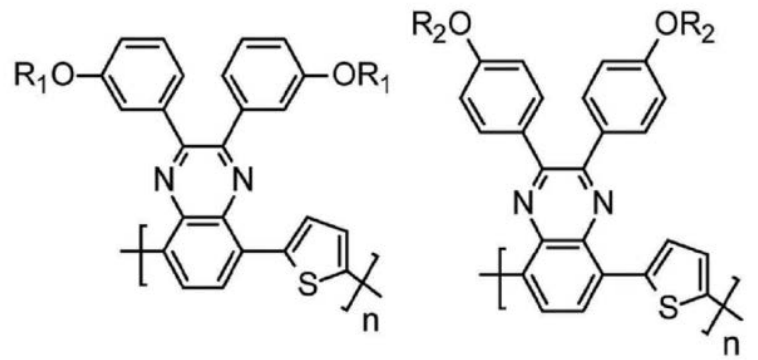

$$
\begin{array}{ll}
T Q_{m 6} & R_{1}=n-C_{6} H_{13} \\
T Q_{m 12} & R_{1}=n-C_{12} H_{25}
\end{array}
$$

$\mathrm{TQ}_{\mathrm{p} 6} \quad \mathrm{R}_{2}=\mathrm{n}-\mathrm{C}_{6} \mathrm{H}_{13}$

\section{PCDTBT}

poly[N-9'-heptadecanyl-2,7-carbazole-alt-5,5-(4',7'-di-2-

thienyl-2', $1^{\prime}, 3^{\prime}$-benzothiadiazole)], poly[[9-(1-octylnonyl)-

9H-carbazole-2,7-diyl]-2,5-thiophenediyl-2,1,3-benzothiadiazole-

4,7-diyl-2,5-thiophenediyl]

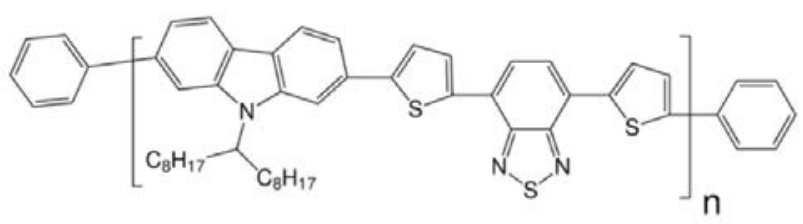




\section{APFO3}

poly[(9,9-dioctylfluorenyl-2,7-diyl)-alt-5,5-(40,70-di-2-thienyl-20,10,30benzothiadiazole)]

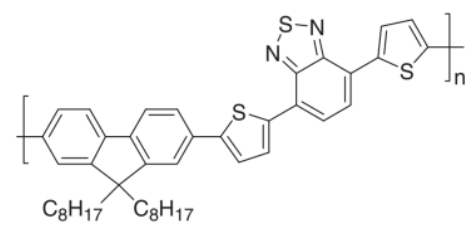

\section{MDMO PPV}

poly[2-methoxy-5-(3',7'-dimethyloctyloxy)-1,4-phenylenevinylene]<smiles>COc1cc(C)c(OCCC(C)CCCC(C)C)cc1C=C(C)C</smiles>

\section{PTB7}

poly(\{4,8-bis[(2-ethylhexyl)oxy]benzo[1,2-b:4,5-b']- dithiophene-2,6-diyl\}\{3-fluoro-2-[(2ethylhexyl)carbonyl]- thieno[3,4-b]thiophenediyl\})<smiles>CCCCC(CC)COC(=O)c1sc(/C=C2\C=C(OCC(CC)CCCC)C(OCC(CC)CCCC)=C2OCC(CC)CCCC)c2sc(C(F)(F)F)c(C)c12</smiles> 


\section{PTB7-Th (PCE10)}

Poly[4,8-bis(5-(2-ethylhexyl)thiophen-2-yl)benzo[1,2-b;4,5-b']dithiophene-2,6-diyl-alt-(4(2-ethylhexyl)-3-fluorothieno[3,4-b]thiophene-)-2-carboxylate-2-6-diyl)]

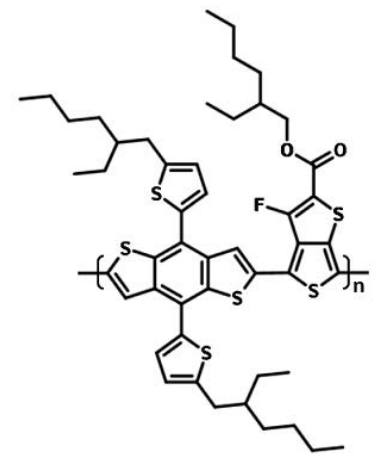

\section{P3HT}

poly(3-hexylthiophene-2,5-diyl)

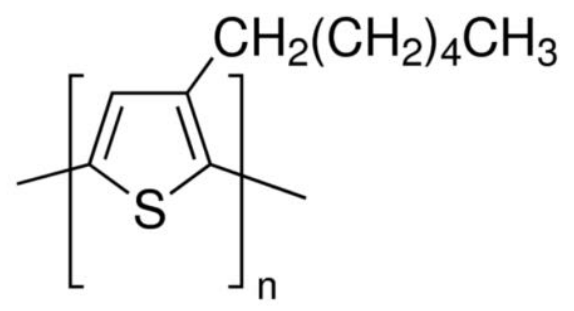

\section{$\mathrm{PC}_{71} \mathrm{BM}$}

[6,6]-phenyl C71 butyric acid methyl ester

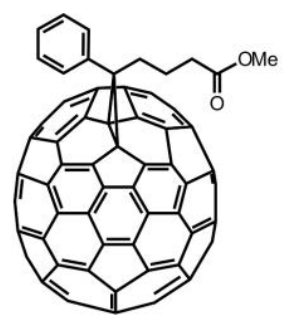




\section{$\mathrm{PC}_{61} \mathrm{BM}$}

[6,6]-phenyl C61 butyric acid methyl ester

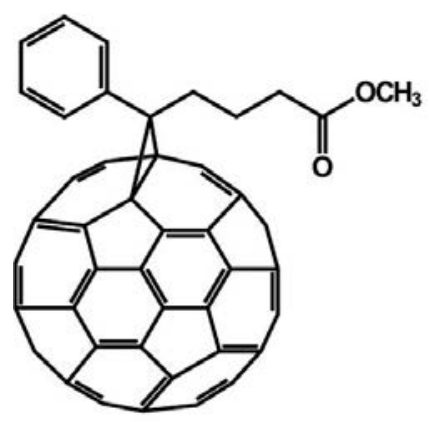

\section{$\mathrm{IC}_{60} \mathrm{BA}$}

$1^{\prime}, 1^{\prime \prime}, 4^{\prime}, 4^{\prime \prime}$-tetrahydro-di[1,4] methanonaphthaleno- $\left[1,2: 2^{\prime}, 3^{\prime}, 56,60: 2^{\prime \prime}, 3^{\prime \prime}\right][5,6]$ fullereneC60

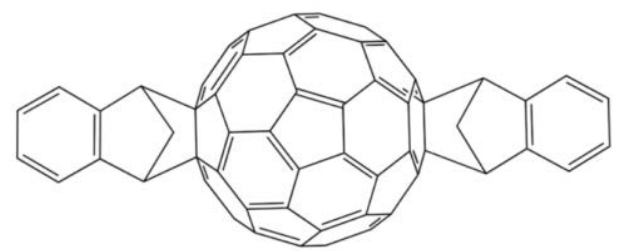

ITIC

3,9-bis(2-methylene-(3-(1,1-dicyanomethylene)-indanone))-5,5,11,11-tetrakis(4hexylphenyl)-dithieno[2,3-d:2',3'-d']-s-indaceno[1,2-b:5,6- $\left.b^{\prime}\right]$ dithiophene

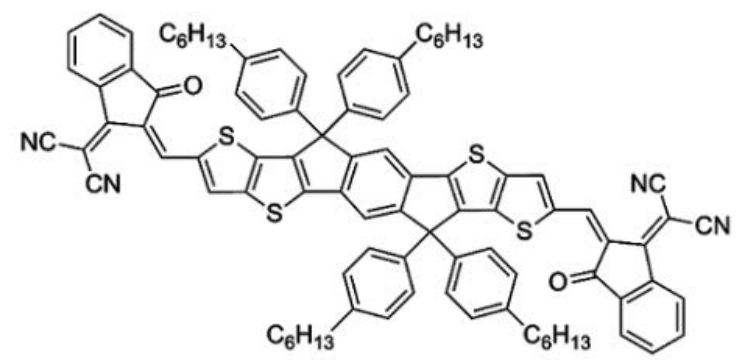




\section{Acknowledgements}

The present thesis would never have been possible without Martijn Kemerink. Thank you Martijn for giving me the opportunity to study under your exemplary supervision in an ideal work environment during the last 4 years. Your inspiring thoughts and meticulous guidance have been a driving force throughout our collaboration and have extensively shaped my professional and personal skills. I really enjoyed our scientific discussions (even the ones I did not completely follow!). The numerous non-professional occasions were also a great pleasure; I pick as highlight the honor of coming and dancing at my wedding!

I would like to thank my co-supervisor Olle Inganäs for all the inspiring talks and constructive feedback throughout my PhD. Thank you Olle for allowing me to work in the BiOrgEl lab and fabricate all organic solar cell devices studied in this thesis.

I would like to express my deepest gratitude to Anthony Turner for recommending me as a PhD candidate to Martijn.

Special thanks go to Armantas Melianas for his mentorship throughout my studies and the creative collaboration in multiple projects. I would also like to thank Ergang Wang for the inspiring work and the high-quality 'Viking' polymers that he supplied. Thank you, Jonas Bergqvist, Luis Ever Aguirre and Yuttapoom Puttisong, for the fast and high-quality work as well as the creative discussions.

In hope of not forgetting someone I would like to thank Deping Qian, Thomas Österberg, Wanzhu Cai, Chuanfei Wang, Yuxin Xia, Qingzhen Bian, Mohammad Javad Jafari, Feng Gao, Niclas Solin, Mats Fahlman and Fengling Zhang for the valuable help in the lab and/or the constructive debates and discussions.

Thank you Anna-Maria Uhlin, Jenny Ählström and Emma Ljungkvist for the all the help with the administrative issues; you made my life easier and mainly focused on science.

It was a great pleasure sharing scientific and personal moments with all the CoMaDeans: Hassan Abdalla, Guangzheng Zuo, Olof Andersson, Indré Urbanavičiūtè, Tim Cornelissen, Davood Abbaszadeh and Tanvi Upreti. Thank you all for the excellent team spirit and for making long office/lab hours tolerable. Thank you Hassan, Guangzheng, Olof and Armantas for the honor of attending my wedding and sharing my happiness (special tribute to Hassan's dancing skills!). 
I would like to thank my brother and my sisters-in-law for all the support throughout my studies as well as my friends from our (aggressively expanding) Greek community in Linköping.

I would not have been able to complete my PhD studies without the support of my beloved wife. Thank you $ү$ ı $\alpha \rho i \mu$ for the unconditional love and understanding, for making sure I show up decently dressed at conferences and for keeping the door open when I returned home from the lab after midnight! People are still discussing the unprecedented dinners you hosted...

I would not be here without the love and personal sacrifices of my parents who gave me the opportunity to study and take a step further in my life. You have set a shining example

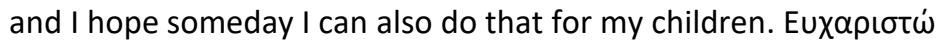

In memory of my late grandmother 


\section{Papers}

The papers associated with this thesis have been removed for copyright reasons. For more details about these see:

http://urn.kb.se/resolve?urn=urn:nbn:se:liu:diva-150998 